Article

\title{
Effects of Carbamazepine, Lacosamide and Zonisamide on Gliotransmitter Release Associated with Activated Astroglial Hemichannels
}

\author{
Kouji Fukuyama, Yuto Ueda and Motohiro Okada * \\ Department of Neuropsychiatry, Division of Neuroscience, Graduate School of Medicine, Mie University, Tsu, \\ Mie 514-8507, Japan; k-fukuyama@clin.medic.mie-u.ac.jp (K.F.); uedayuto@gmail.com (Y.U.) \\ * Correspondence: okadamot@clin.medic.mie-u.ac.jp; Tel.: +81-59-231-5018
}

Received: 5 May 2020; Accepted: 3 June 2020; Published: 5 June 2020

check for updates

\begin{abstract}
Recent studies using the genetic partial epilepsy model have demonstrated that hyperfunction of astroglial hemichannels contributes to pathomechanism of epileptic seizure. Therefore, to explore the novel anticonvulsive mechanisms, the present study determined the effects of voltage-dependent $\mathrm{Na}^{+}$channel (VDSC)-inhibiting anticonvulsants, carbamazepine (CBZ), lacosamide (LCM), and zonisamide (ZNS) on the astroglial release of L-glutamate and adenosine triphosphate (ATP). The effects of subchronic administration of therapeutic-relevant dose of three anticonvulsants on the release of L-glutamate and ATP in the orbitofrontal cortex (OFC) were determined using microdialysis. The concentration-dependent effects of acute and subchronic administrations of anticonvulsants on astroglial gliotransmitter release were determined using primary cultured astrocytes. The concentration-dependent effects of subchronic administrations of anticonvulsants on connexin43 (Cx43) expression in the plasma membrane of primary cultured astrocytes were determined using the Simple Western system. An increase in the levels of extracellular $\mathrm{K}^{+}$resulted in a concentration-dependent increase in the astroglial release of L-glutamate and ATP. The depleted levels of extracellular $\mathrm{Ca}^{2+}$ alone did not affect astroglial gliotransmitter release but did accelerate $\mathrm{K}^{+}$-evoked gliotransmitter release via activation of astroglial hemichannels. Both non-selective hemichannel inhibitor carbenoxolone (CBX) and selective Cx43 inhibitor GAP19 prevented both gliotransmitter release through activated astroglial hemichannels and the hemichannel-activating process induced by elevation of the levels of extracellular $\mathrm{K}^{+}$with depletion of the levels of extracellular $\mathrm{Ca}^{2+}$. ZNS subchronically decreased Cx43 expression and acutely/subchronically inhibited Cx43 hemichannel activity. LCM acutely inhibited hemichannel activity but did not subchronically affect Cx43 expression. Therapeutic-relevant concentration of CBZ did not affect hemichannel activity or Cx43 expression, but supratherapeutic concentration of CBZ decreased Cx43 expression and hemichannel activity. Therefore, the present study demonstrated the distinct effects of CBZ, LCM, and ZNS on gliotransmitter release via modulation of astroglial hemichannel function. The different features of the effects of three VDSC-inhibiting anticonvulsants on astroglial transmission associated with hemichannels, at least partially, possibly contributing to the formation of the properties of these three anticonvulsants, including the antiepileptic spectrum and adverse effects regarding mood and cognitive disturbance.
\end{abstract}

Keywords: astrocyte; carbamazepine; lacosamide; zonisamide; microdialysis; hemichannel

\section{Introduction}

The principle antiepileptic mechanism of many first-line anticonvulsants against partial epilepsy, including carbamazepine (CBZ), lacosamide (LCM), and zonisamide (ZNS), is considered to be 
use-dependent inhibition of voltage-dependent $\mathrm{Na}^{+}$channel (VDSC) activity [1]; however, the clinical features of VDSC-inhibiting anticonvulsants cannot be fully understood solely from the VDSC inhibition profile. Indeed, LCM has a more favourable cognitive profile compared to CBZ and ZNS [2,3]. Both CBZ and ZNS enhance fast inactivation without affecting the slow inactivation phases of VDSC, whereas LCM enhances slow inactivation without affecting the fast inactivation phases [4]. These differences in the VDSC inhibition profiles among the three anticonvulsants may distinguish LCM from CBZ and ZNS in terms of cognitive function. Phenytoin and lamotrigine enhance both the slow and fast VDSC inactivation phases [4]; however, phenytoin and lamotrigine negatively and positively affect cognitive function, respectively [5]. Therefore, these clinical and preclinical findings suggest that the clinical spectrum of each anticonvulsant should be considered as being constituted by the integration between the inhibition of spreading epileptic hyperexcitability via VDSC inhibition with effects on other transmission regulating systems [1,6].

In the past two decades, molecular biological and pharmacological studies have gradually been revealing that the complex mechanisms of the development of epileptogenesis/ictogenesis comprise the acquisition of everlasting epilepsy-specific transmission abnormality induced by genetic abnormality with event-related transmission abnormality [7-13]. Recently, we have demonstrated the pathomechanism and pathophysiology of autosomal-dominant sleep-related hypermotor epilepsy (ADSHE) [14] with S284L mutation in the CHRNA4 gene encoding the $\alpha 4$ subunit of the nicotinic acetylcholine receptor (nAChR) [7-10]. ADSHE (previously known as autosomal-dominant nocturnal frontal lobe epilepsy) was first identified as a distinct familial idiopathic epilepsy in 1994 [15]. Although, typically, ADSHE seizures are symptomatically comparable to those seen in frontal lobe epilepsy and occur predominantly during non-rapid eye movement sleep $[14,16,17]$, numerous missense mutations in various genes have been identified in many ADSHE pedigrees [14,17]. Usually, the majority of ADSHE seizures are well controlled by CBZ, which leads to remission in approximately $50-60 \%$ of ADSHE patients [16,18]; however, ADSHE patients with a CBZ-resistant feature require other anticonvulsants such as ZNS and LCM [17-19]. Furthermore, ADSHE seizures are the sole major symptom of ADSHE patients, and additional neuropsychiatric disturbances have been reported in lower than 3\% of ADSHE patients [14]; however, ADSHE patients with an S284L-mutation have been known to be comorbid with a cognitive deficits such as schizophrenia-like psychosis, autism spectrum disorder, and intellectual disability [17,19].

Our recent studies using a genetic animal model with rats, namely S286L-TG, bearing the missense S286L-mutation of rat Chrna4 that corresponds to an S284L-mutation in human CHRNA4, demonstrated several pathomechanisms and pathophysiologies of ADSHE seizures with an S284L-mutation [7-10]. Primarily, the impaired excitatory function of S286L-mutant $\alpha 4 \beta 2$-nAChR generates relatively GABAergic disinhibition in intrathalamic circuits, resulting in hyperexcitable glutamatergic transmission in both the thalamocortical motor and thalamic hyperdirect pathways [7-10]. Additionally, S286L-mutant $\alpha 4 \beta 2$-nAChR creates deficits in inhibitory regulation on connexin 43 (Cx43) expression in the astroglial plasma membrane [8,9]. Based on these functional abnormalities, including the intrathalamic circuits, thalamocortical motor, thalamic hyperdirect pathways, and the physiological bursts, sleep spindle during non-rapid eye movement sleep possibly activate upregulated Cx43 hemichannel activity, leading to the generation of ADSHE focus in the frontal cortex [8]. We also demonstrated the three candidate pathophysiologies of CBZ-resistant/ZNS-sensitive ADSHE seizures with S284L-mutation. ZNS compensates the GABAergic disinhibition induced by impaired S286L-mutant $\alpha 4 \beta 2-n A C h R$ in the focus region [7]. ZNS inhibits the thalamic hyperdirect pathway by enhancing the endogenous group II metabotropic glutamate receptor, xanthurenic acid, resulting in the prevention of nocturnal paroxysmal dystonia, an ADSHE paroxysmal movement disorder $[10,20]$. Furthermore, chronic administration of ZNS prevents ADSHE focus generation due to reduction of astroglial Cx43 expression on the plasma membrane in the focus region [8].

Cx43 is the most predominant expression connexin isoform in astrocytes [21]. Six connexins assemble to form a homomeric/heteromeric connexon. Two connexon in two neighbouring cells 
(including neurons, astrocytes, oligodendrocytes, and microglia) form a gap-junction with an aqueous pore and charged surface walls, but a single connexon contributes to the chemical connection between the intracellular and extracellular spaces as a hemichannel [21]. Gap-junctions and hemichannels are crucial to the coordination/maintenance of physiologic activity, including neuronal excitability, synaptic plasticity, tripartite synaptic transmission, and homeostasis maintenance in the central nervous system [21]. Astroglial hemichannels also regulate ionic homeostasis, including ion movement regulation between intracellular and extracellular spaces, and the release of several gliotransmitters, including adenosine triphosphate (ATP), nicotinamide adenine dinucleotide, L-glutamate, or prostaglandins, involved in autocrine/paracrine signalling [22]. During the resting stage, astroglial hemichannels do not contribute to gliotransmitter release due to their low opening probability [22,23]; however, activation of hemichannel induced by plasma membrane depolarisation and extracellular/intracellular cation mobilization (i.e., elevations of extracellular $\mathrm{K}^{+}$and intracellular $\mathrm{Ca}^{2+}$ with a reduction of extracellular $\mathrm{Ca}^{2+}$ ) generates gliotransmitter release through activated hemichannels [22,23]. It has also been well established that astrocytes play important roles in the development of epileptogenesis under subclinical pro-inflammatory responses [21,24]. Furthermore, Cx43 is involved in memory formation under physiological function, whereas both impaired and hyperactivated astroglial function generate cognitive disturbance [25].

The expression of Cx43 is increased in glia but not in neurons in pentylenetetrazole kindled rats [26], as well as in patients with refractory temporal lobe epilepsy and type IIB focal cortical dysplasia [27,28]. Furthermore, the upregulation of Cx43 in S286L-TG has also been demonstrated [8,9]. In particular, the inhibitors of connexin channels can prevent the onset of epileptic seizures [29]. These findings suggest that inhibition of the functional connexin expression in the plasma membrane with hemichannel activity can prevent the astroglial processes of epileptic seizures. Furthermore, we have already hypothesised that the interplay between astroglial depolarization and hemichannel activation produces a further astroglial regenerative activation circular sequence: plasma membrane depolarization activates an accelerated increase in the movements of cations via activated hemichannels, resulting in further depolarization of the astroglial membrane potential $[8,9,30]$. Therefore, astroglial hemichannel inhibition is a reasonable candidate for anticonvulsive medication; however, considering the effects of astroglial hemichannel inhibition on cognition [25] is important for ensuring the quality of life (QOL) of patients with epilepsy. We have also reported that upregulated Cx43 and/or astroglial hemichannels play important roles in the pathophysiology of CBZ-resistant and ZNS-sensitive epileptic seizures of S286L-TG [8,9]. Furthermore, several clinical studies have reported that LCM can suppress CBZ-resistant ADSHE seizures [18,19]. Therefore, we hypothesise that functional abnormality regarding astroglial hemichannels provides the pathophysiology of CBZ-resistant epileptic seizures. According to our hypothesis, in the present study, to explore the effects of VDSC-inhibiting anticonvulsants CBZ, LCM, and ZNS on astroglial hemichannel expression and activity, we determined the concentration-dependent effects of CBZ, LCM, and ZNS on Cx43 expression in the plasma membrane, and astroglial transmission-associated activated hemichannels using microdialysis, primary cultured astrocytes, and the Simple Western system.

\section{Results}

\subsection{Effects of the Levels of Extracellular $\mathrm{Ca}^{2+}$ and $\mathrm{K}^{+}$on the Astroglial Release of $\mathrm{L}$-Glutamate and ATP (Study_1)}

Electrophysiological study demonstrated that decreased levels of extracellular $\mathrm{Ca}^{2+}$ and increased extracellular $\mathrm{K}^{+}$activate hemichannel activity [22,23,31]. To study gliotransmitter release through astroglial hemichannels, the interaction between extracellular cations and hemichannel inhibitors on the astroglial release of L-glutamate and ATP was determined using primary cultured astrocytes. To determine the effects of hemichannel inhibitors, carbenoxolone (CBX; a non-selective inhibitor) [30,32] and GAP19 (a selective Cx43 inhibitor) [30,32] on the astroglial release of L-glutamate and ATP 
induced by $\mathrm{Ca}^{2+}$-free (FC-ACSF), $100 \mathrm{mM} \mathrm{K}^{+}$(HK-ACSF) or $\mathrm{Ca}^{2+}$-free with $100 \mathrm{mM} \mathrm{K}^{+}$(FCHK-ACSF) containing artificial cerebrospinal fluid (ACSF), during the 21 days after culture (DIV21) to DIV28, the astrocytes were incubated in Dulbecco's modified Eagle's medium containing $10 \%$ fetal calf serum (fDMEM) without any agents. On DIV28, after being washed out, during pretreatment incubation, the astrocytes were incubated in ACSF with or without CBX $(100 \mu \mathrm{M})$ or GAP19 $(20 \mu \mathrm{M})[8,30]$ for $20 \mathrm{~min}$. After pretreatment incubation, the astrocytes were incubated in FC-ACSF, HK-ACSF, or FCHK-ACSF containing the same agent of pretreatment for $20 \mathrm{~min}$.

Study_1 indicates the statistical significance regarding the interaction between the extracellular cation levels and the hemichannel inhibitors on the astroglial release of L-glutamate $\left(\mathrm{F}_{\text {ion }}(3,45)=\right.$ 62.9, $p<0.01 ; \mathrm{F}_{\text {agent }}(2,15)=2.8,>0.05$; and $\left.\mathrm{F}_{\text {ionagent }}(6,45)=7.2, p<0.01\right)$ and ATP $\left(\mathrm{F}_{\text {ion }}(3,45)=\right.$ 157.6, $p<0.01 ; \mathrm{F}_{\text {agent }}(2,15)=9.1, p<0.01$; and $\mathrm{F}_{\text {ion }}{ }^{*}$ agent $\left.(6,45)=13.8, p<0.01\right)$. Extracellular $\mathrm{Ca}^{2+}$-free (incubated in FC-ACSF for $20 \mathrm{~min}$ ) did not affect the astroglial release of L-glutamate or ATP compared to those in ACSF (Figure 1A,B). Increased extracellular $\mathrm{K}^{+}(100 \mathrm{mM}$ ) (incubated in HK-ACSF for $20 \mathrm{~min}$ ) enhanced the astroglial release of $\mathrm{L}$-glutamate and ATP (Figure 1A,B). Extracellular $\mathrm{Ca}^{2+}$-free with $100 \mathrm{mM} \mathrm{K}^{+}$condition (incubated in FCHK-ACSF for $20 \mathrm{~min}$ ) drastically increased the astroglial release of L-glutamate and ATP (Figure 1A,B). Cx43 hemichannel inhibitor GAP19 $(20 \mu \mathrm{M})$ and non-selective hemichannel inhibitor CBX $(100 \mu \mathrm{M})$ suppressed the HK-ACSF- and FCHK-ACSF-evoked release of L-glutamate and ATP (Figure 1A,B). The results in Study_1 indicate that astroglial hemichannels are non-functional during resting stage. A decrease in extracellular $\mathrm{Ca}^{2+}$ alone cannot activate astroglial hemichannels but can accelerate the function of activated hemichannels by elevation of the level of extracellular $\mathrm{K}^{+}$.
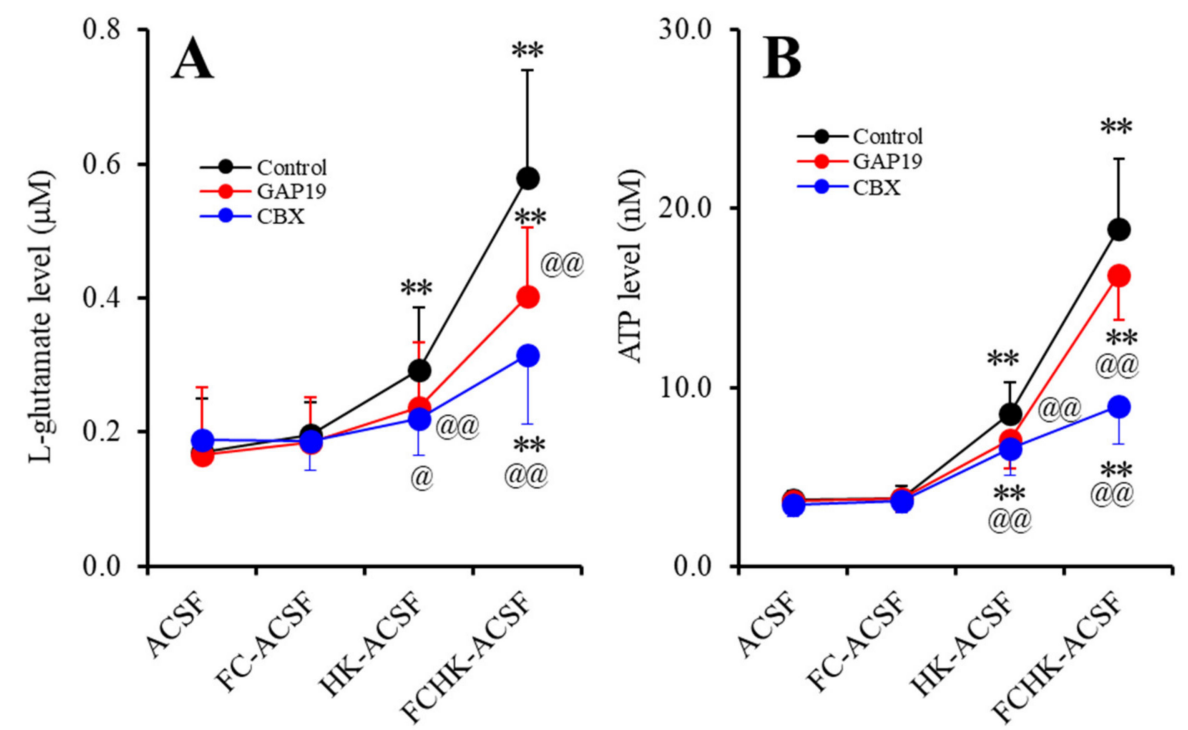

Figure 1. Effects of the extracellular $\mathrm{Ca}^{2+}$ and $\mathrm{K}^{+}$and hemichannel inhibitors carbenoxolone (CBX; a non-selective inhibitor, $100 \mu \mathrm{M}$ ) and GAP19 (a selective Cx43 inhibitor, $20 \mu \mathrm{M}$ ) on the astroglial release of (A) L-glutamate and (B) adenosine triphosphate (ATP). Primary cultured astrocytes were incubated in artificial cerebrospinal fluid (ACSF), $\mathrm{Ca}^{2+}$-free ACSF (FC-ACSF), $\mathrm{K}^{+}$-containing ACSF (HK-ACSF; $100 \mathrm{mM}$ ), and $\mathrm{Ca}^{2+}$-free with $100 \mathrm{mM} \mathrm{K}{ }^{+}$-containing ACSF (FCHK-ACSF) for $20 \mathrm{~min}$. Ordinate: mean \pm standard deviation (SD) $(n=6)$ of the extracellular levels of L-glutamate $(\mu \mathrm{M})$ and ATP (nM). ${ }^{* *} p<0.01$ relative to ACSF, and ${ }^{\circledR} p<0.05$ and ${ }^{\circledR @} p<0.01$ relative to the control (without hemichannel inhibitors) by MANOVA with Tukey's post-hoc test.

\subsection{Effects of the Hemichannel Inhibitors on the Repetitive FCHK-ASCF-Evoked Astroglial Release of L-Glutamate and ATP}

It is well known that during the resting stage, hemichannels have a low opening probability, but under extracellular cation conditions, increased $\mathrm{K}^{+}$and decreased $\mathrm{Ca}^{2+}$ levels activate hemichannel 
activity [22,23]. A previous microdialysis study demonstrated that repetitive $100 \mathrm{mM} \mathrm{K} \mathrm{K}^{+}$-evoked stimulation increased L-glutamate release via activated astroglial hemichannels in a use-dependent manner [8]. In a previous study using primary cultured astrocytes, $100 \mathrm{mM} \mathrm{K}{ }^{+}$-evoked stimulation generated the activation of astroglial hemichannels, but $50 \mathrm{mM} \mathrm{K}^{+}$-evoked stimulation did not [30]. Therefore, to study the mechanisms of use-dependent repetitive $\mathrm{K}^{+}$-evoked gliotransmitter release, the effects of $20 \mu \mathrm{M}$ GAP19 and $100 \mu \mathrm{M} \mathrm{CBX}$ on the astroglial release of L-glutamate and ATP induced by repetitive FCHK-ACSF-evoked stimulation were determined.

2.2.1. Effects of GAP19 and Carbenoxolone (CBX) on the FCHK-ACSF-Evoked Astroglial Release of L-Glutamate and ATP through Activated Hemichannels (Study_2)

To study the effects of the hemichannel inhibitors on gliotransmitter release through activated hemichannels, after the first FCHK-ACSF-evoked stimulation for $20 \mathrm{~min}$, the astrocytes were incubated in ACSF containing $100 \mu \mathrm{M} \mathrm{CBX}$ or $20 \mu \mathrm{M}$ GAP19 for $20 \mathrm{~min}$ (post-treatment). After the post-treatment, the astrocytes were incubated in FCHK-ACSF containing the same agent for $20 \mathrm{~min}$ again (the second FCHK-ACSF-evoked stimulation). The schematic experimental design of Study_2 is represented in Figure 2C.

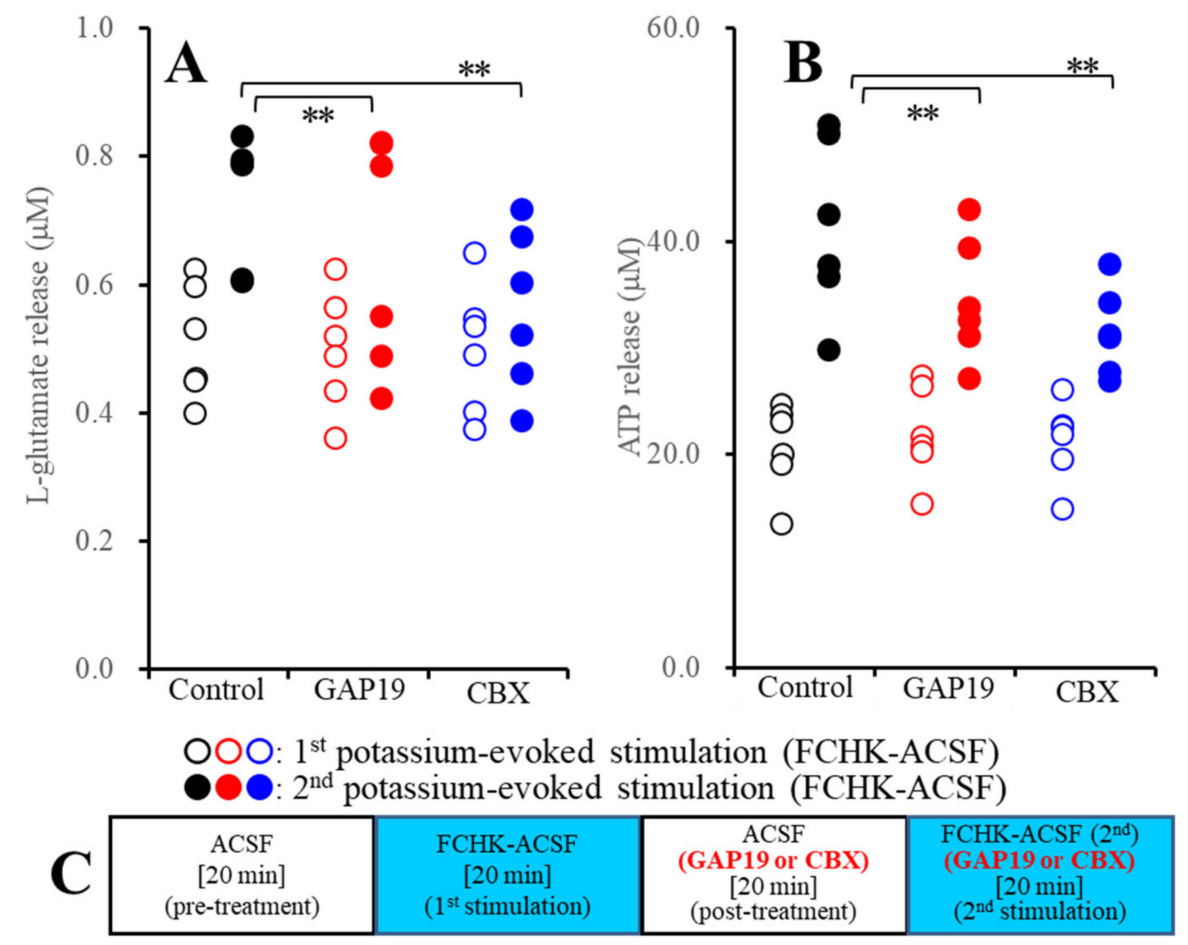

Figure 2. Effects of the hemichannel inhibitors (20 $\mathrm{MM}$ GAP19 and $100 \mu \mathrm{M}$ CBX) on the astroglial release of (A) L-glutamate, (B) ATP induced by FCHK-ACSF associated with activated astroglial hemichannels, and $(C)$ the experimental design. Ordinate: mean \pm SD $(n=6)$ of the levels of L-glutamate $(\mu \mathrm{M})$ and ATP $(\mathrm{nM}) .{ }^{* *} p<0.01$ relative to the control by two-way ANOVA with Tukey's post-hoc test.

Study_2 indicates the statistical significance regarding the interaction between the extracellular cation levels and the hemichannel inhibitors on the astroglial release of L-glutamate $\left(\mathrm{F}_{\text {event }}(2,20)\right.$ $=13.3, p<0.01 ; \mathrm{F}_{\mathrm{Cx} 43}(1,10)=4.9, p>0.05$; and $\left.\mathrm{F}_{\text {event }{ }^{*} \mathrm{C} \times 43}(2,20)=10.6, p<0.01\right)$ and ATP $\left(\mathrm{F}_{\text {event }}\right.$ $(2,20)=19.8, p<0.01 ; \mathrm{F}_{\mathrm{Cx} 43}(1,10)=24.1, p<0.01$; and $\left.\mathrm{F}_{\text {event }{ }^{*} \mathrm{Cx} 43}(2,20)=27.3, p<0.01\right)$. Both CBX and GAP19 inhibited the second FCHK-ACSF-evoked astroglial release of L-glutamate and ATP (Figure 2A,B). The results of Study_2 indicate that both CBX and GAP19 inhibit astroglial release through activated hemichannels. 
2.2.2. Effects of GAP19 and CBX on the Repetitive FCHK-ACSF-Evoked Astroglial Release of L-Glutamate and ATP through Unactivated and Activated Hemichannels (Study_3)

To study the effects of the hemichannel inhibitors on the activation-process of astroglial hemichannels, during Study_3, primary cultured astrocytes were incubated in ACSF or FCHK-ACSF without (control) or with $100 \mu \mathrm{M}$ CBX or $20 \mu \mathrm{M}$ GAP19. The schematic experimental design of Study_3 is represented in Figure 3C. Before the first stimulation, the astrocytes were incubated in ACSF containing $100 \mu \mathrm{M}$ CBX or $20 \mu \mathrm{M}$ GAP19 for $20 \mathrm{~min}$ (pretreatment), and after pretreatment, the astrocytes were evoked by FCHK-ACSF containing the same hemichannel inhibitor for 20 min (the first FCHK-ACSF stimulation). After the post-treatment in the same medium of pretreatment, the astrocytes were also incubated in FCHK-ACSF containing the same agent for 20 min again (the second FCHK-ACSF-evoked stimulation).

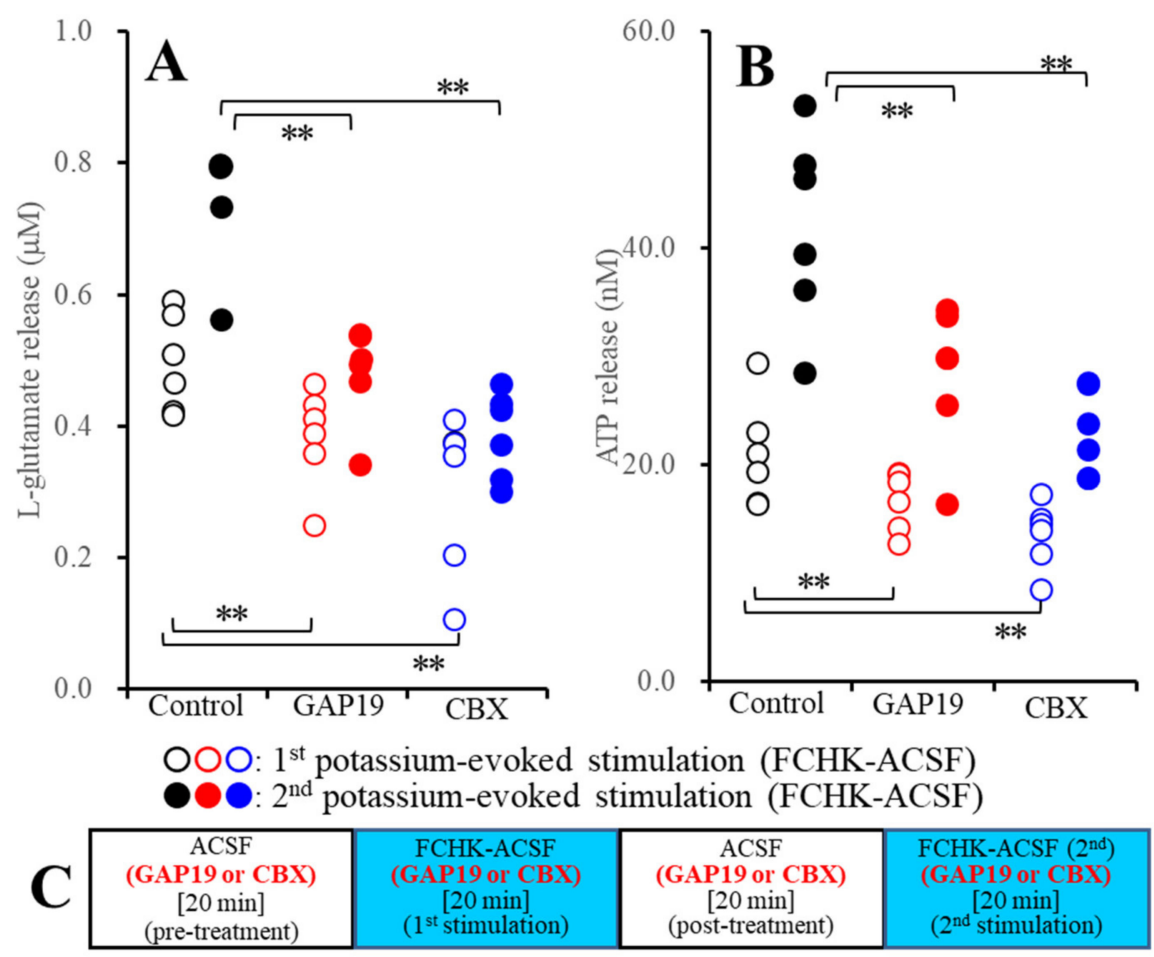

Figure 3. Effects of the hemichannel inhibitors $(20 \mu \mathrm{M}$ GAP19 and $100 \mu \mathrm{M} \mathrm{CBX})$ on the repetitive $\mathrm{K}^{+}$-evoked (FCHK-ACSF) astroglial release of (A) L-glutamate, (B) ATP, and (C) the experimental design. Ordinate: mean $\pm \mathrm{SD}(n=6)$ of the levels of L-glutamate $(\mu \mathrm{M})$ and $\operatorname{ATP}(\mathrm{nM}) .{ }^{* *} p<0.01$ relative to the control by two-way ANOVA with Tukey's post-hoc test.

Study_3 indicates the statistical significance regarding the interaction between the extracellular cation levels and the hemichannel inhibitors on the astroglial release of L-glutamate $\left(\mathrm{F}_{\text {event }}(2,20)\right.$ $=73.1, p<0.01 ; \mathrm{F}_{\mathrm{Cx} 43}(1,10)=5.0, p<0.05 ;$ and $\left.\mathrm{F}_{\text {event }}{ }^{*} \mathrm{Cx} 43(2,20)=11.7, p<0.01\right)$ and ATP $\left(\mathrm{F}_{\text {event }}\right.$ $(2,20)=88.3, p<0.01 ; \mathrm{F}_{\mathrm{Cx} 43}(1,10)=22.7, p<0.01 ;$ and $\left.\mathrm{F}_{\text {event }}{ }^{*} \mathrm{Cx} 43(2,20)=18.3, p<0.01\right)$. After the application of $100 \mu \mathrm{M}$ CBX and $20 \mu \mathrm{M}$ GAP19 to unactivated hemichannels, both CBX and GAP19 reduced the first FCHK-ACSF-evoked release of l-glutamate and ATP (Figure 3A,B). Similar to the first FCHK-ACSF-evoked stimulation, both CBX and GAP19 reduced the second FCHK-ACSF-evoked release of 1-glutamate and ATP (Figure 3A,B). Taken together with the results of Study_2, both CBX and GAP19 suppress not only the astroglial release through activated hemichannels, but also the activation process of the astroglial hemichannels. 
2.3. Concentration-Dependent Effects of Carbamazepine (CBZ), Lacosamide (LCM), and Zonisamide (ZNS) on the Astroglial Release of L-Glutamate and ATP (Study_4)

To study the concentration-dependent effects of the subchronic administration of CBZ, LCM, and ZNS on the basal astroglial release of L-glutamate and ATP, during DIV21 to DIV28, the astrocytes were incubated in fDMEM containing $\operatorname{CBZ}(0,10,30$, or $100 \mu \mathrm{M}), \operatorname{LCM}(0,10,30$, or $100 \mu \mathrm{M})$ or ZNS $(0,30$, 100 , or $300 \mu \mathrm{M}$ ) for 7 days. On DIV28, after being washed out, during the pretreatment, the astrocytes were incubated in ACSF containing the same agent for $20 \mathrm{~min}$. To study the concentration-dependent effects of the acute administration of CBZ, LCM, and ZNS on the basal astroglial release of L-glutamate and ATP, during DIV21 to DIV28, the astrocytes were incubated in fDMEM without anticonvulsant for 7 days. On DIV28, after being washed out, during the pretreatment, the astrocytes were incubated in ACSF with or without CBZ $(10,30$, or $100 \mu \mathrm{M})$, LCM $(10,30$, or $100 \mu \mathrm{M})$ or ZNS $(30,100$, or $300 \mu \mathrm{M})$ for $20 \mathrm{~min}$.

Neither acute nor subchronic administration of CBZ $(10,30$, or $100 \mu \mathrm{M})$, LCM $(10$, 30, or $100 \mu \mathrm{M})$, or ZNS $(30,100$, or $300 \mu \mathrm{M})$ affected the basal astroglial release of L-glutamate and ATP (Figure 4).
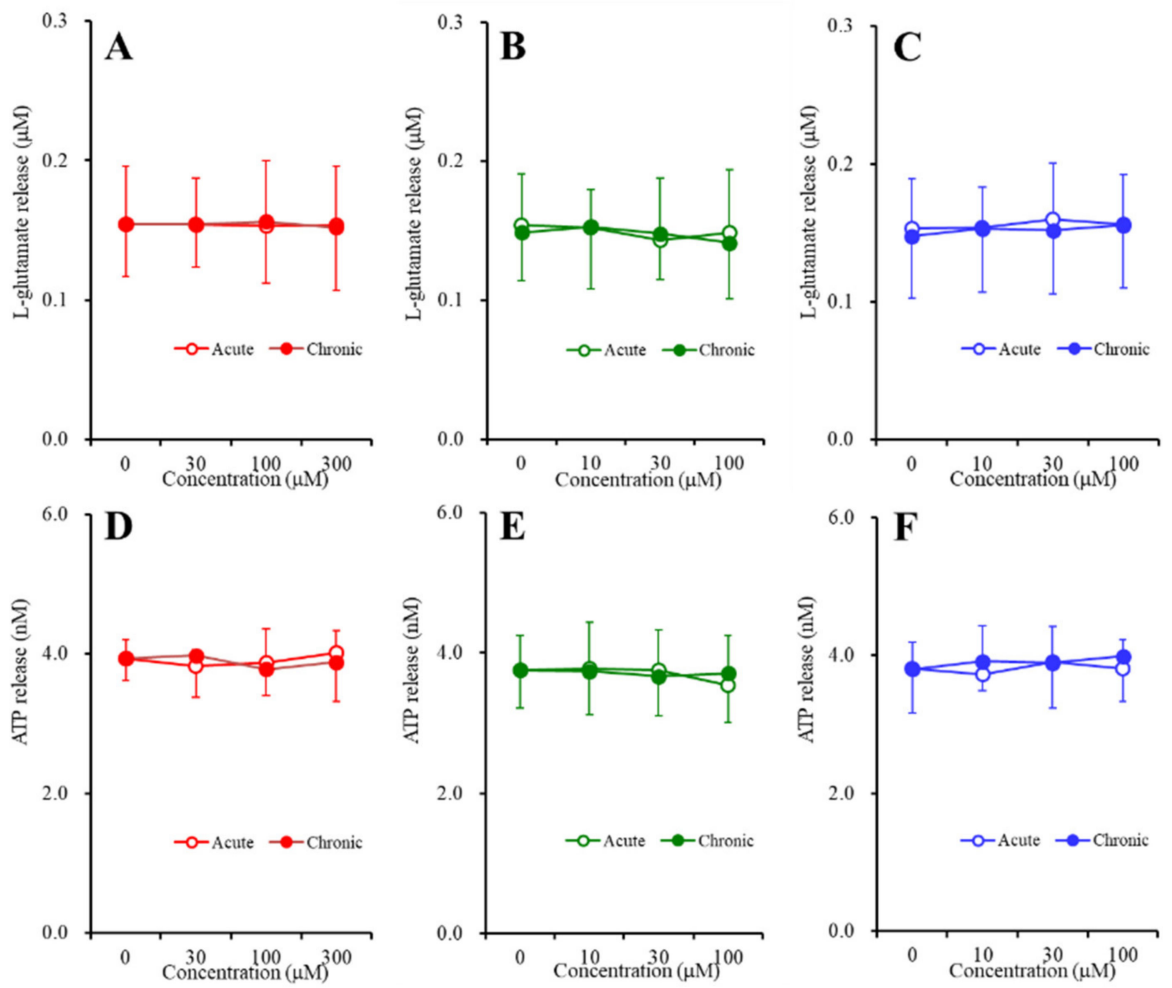

Figure 4. Concentration-dependent effects of the acute (open circles) and subchronic (closed circles) administration of (A,D) zonisamide (ZNS) (30, 100, or $300 \mu \mathrm{M}),(\mathbf{B}, \mathbf{E})$ carbamazepine (CBZ) (10, 30, or $100 \mu \mathrm{M})$, and $(\mathbf{C}, \mathbf{F})$ lacosamide $(\mathrm{LCM})(10,30$, or $100 \mu \mathrm{M})$ on the basal astroglial release of $(\mathbf{A}-\mathrm{C})$ L-glutamate and (D-F)ATP. Ordinate: mean $\pm \mathrm{SD}(n=6)$ of the basal astroglial release of L-glutamate $(\mu \mathrm{M})$ and ATP $(\mathrm{nM})$. Abscissa: concentration of ZNS, CBZ, and LCM $(\mu \mathrm{M})$.

To study the effects of the acute and subchronic administration of CBZ, LCM, and ZNS on the astroglial release of L-glutamate and ATP induced by repetitive FCHK-ACSF-evoked stimulation, after the above sampling of the basal release, the astrocytes were incubated in FCHK-ACSF containing the same agent for $20 \mathrm{~min}$ (the first stimulation). After the first stimulation, the incubation medium was switched to ACSF containing the same agent (post-treatment) for $20 \mathrm{~min}$, and then the astrocytes were evoked by FCHK-ACSF containing the same agent for $20 \mathrm{~min}$ (the second stimulation) again. The F-values by MANOVA are indicated in the legend of Figure 5. 

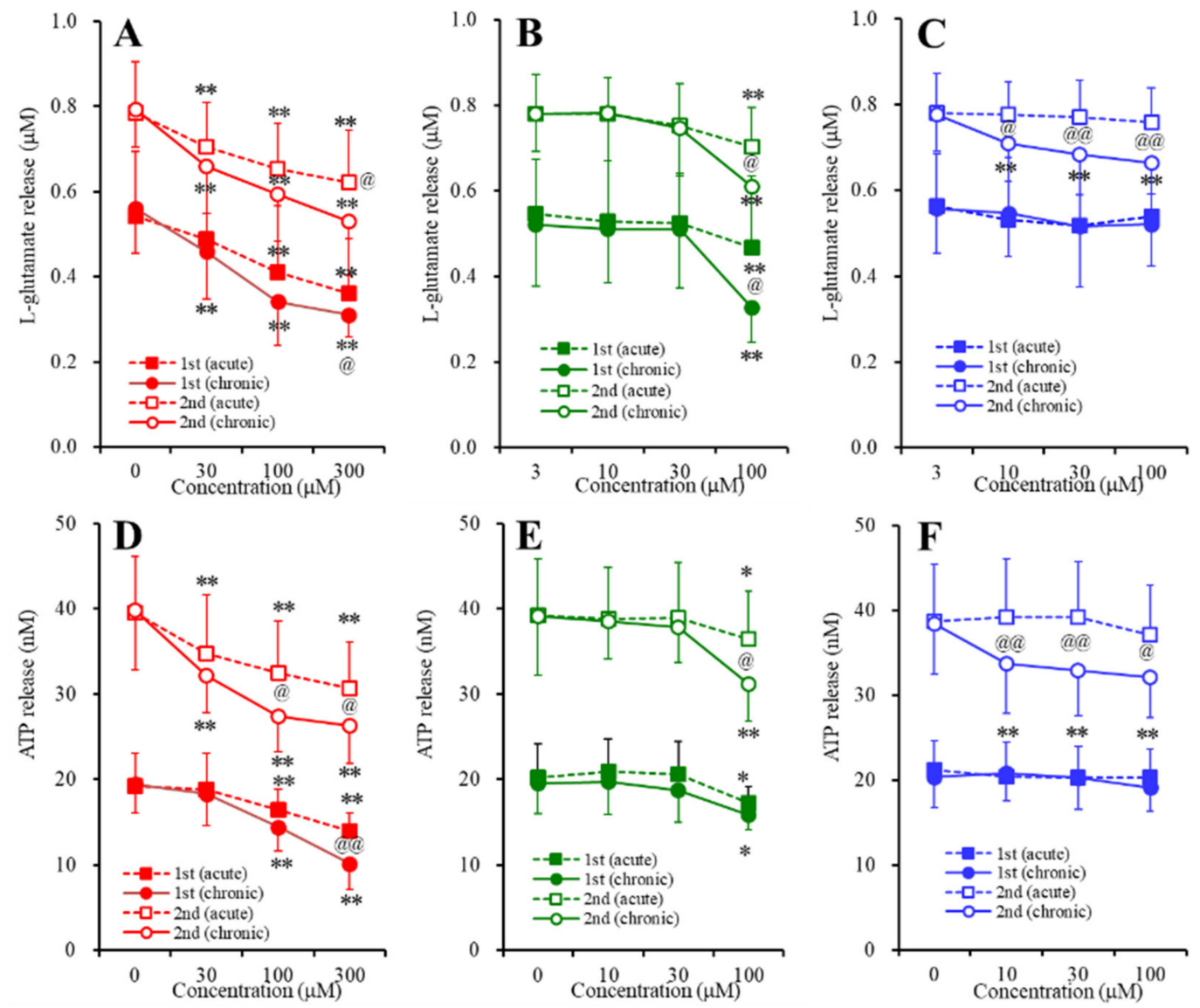

Figure 5. Concentration-dependent effects of the acute (squares) and subchronic (circles) administration of (A,D) ZNS (30, 100, or $300 \mu \mathrm{M}),(\mathbf{B}, \mathbf{E})$ CBZ $(10,30$, or $100 \mu \mathrm{M})$, and (C,F) LCM (10, 30, or $100 \mu \mathrm{M})$ on the repetitive $\mathrm{K}^{+}$-evoked (first: closed marks; second: opened marks) astroglial release of (A-C) L-glutamate and (D-F) ATP. Ordinate: mean \pm SD $(n=6)$ of the astroglial release of L-glutamate $(\mu \mathrm{M})$ and ATP (nM). Abscissa: concentration of ZNS, CBZ, and LCM $(\mu \mathrm{M}) .{ }^{*} p<0.05$ and ${ }^{* *} p<0.01$ relative to the anticonvulsant-free condition, and ${ }^{@} p<0.05$ and ${ }^{@ @ ~} p<0.01$ relative to acute administration by MANOVA with Tukey's multiple comparison. The F-values of the effects of ZNS on L-glutamate and ATP were $\mathrm{F}_{\mathrm{ZNS}}(3,30)=77.4(p<0.01), \mathrm{F}_{\text {event }}(1,10)=147.6(p<0.01), \mathrm{F}_{\text {treatment }}(1,10)=0.4(p>0.05)$, $\mathrm{F}_{Z N S^{*} \text { treatment }}(3,30)=3.2(p<0.05), \mathrm{F}_{\text {event }}{ }^{*}$ treatment $(1,10)=0.2(p>0.05), \mathrm{F}_{Z N S^{*} \text { event }}(3,30)=0.5(p>0.05)$, and $\mathrm{F}_{\mathrm{ZNS}{ }^{*} \text { event }}$ treatment $(3,30)=0.2(p>0.05)$, and on ATP were $\mathrm{F}_{\mathrm{ZNS}}(3,30)=167.9(p<0.01), \mathrm{F}_{\text {event }}(1,10)$ $=422.5(p<0.01), \mathrm{F}_{\text {treatment }}(1,10)=0.8(p>0.05), \mathrm{F}_{Z N S^{*} \text { treatment }}(3,30)=10.2(p<0.01), \mathrm{F}_{\text {event }}{ }^{*}$ treatment $(1,10)=0.7(p>0.05), \mathrm{F}_{Z N S^{*} \text { event }}(3,30)=19.1(p<0.01)$, and $\mathrm{F}_{Z N S^{*} \text { event }}{ }^{*}$ treatment $(3,30)=1.3(p>0.05)$, respectively. The F-values of the effects of CBZ on L-glutamate and ATP were $\mathrm{F}_{\mathrm{CBZ}}(3,30)=64.3$ $(p<0.01), \mathrm{F}_{\text {event }}(1,10)=402.2(p<0.01), \mathrm{F}_{\text {treatment }}(1,10)=0.4(p>0.05), \mathrm{F}_{\mathrm{CBZ}}{ }^{*}$ treatment $(3,30)=12.4$ $(p<0.01), \mathrm{F}_{\text {event }}^{*}$ treatment $(1,10)=1.1(p>0.05), \mathrm{F}_{\mathrm{CBZ}}{ }^{*}$ event $(3,30)=0.5(p>0.05)$, and $\mathrm{F}_{\mathrm{CBZ}^{*} \text { event }}{ }^{*}$ treatment $(3,30)=0.2(p>0.05)$, and on ATP were $\mathrm{F}_{\mathrm{CBZ}}(3,30)=26.9(p<0.01), \mathrm{F}_{\text {event }}(1,10)=388.0(p<0.01)$, $\mathrm{F}_{\text {treatment }}(1,10)=0.4(p>0.05), \mathrm{F}_{C B Z^{*} \text { treatment }}(3,30)=8.7(p>0.05), \mathrm{F}_{\text {event treatment }}(1,10)=0.1(p>0.05)$, $\mathrm{F}_{\mathrm{CBZ} \text { event }}(3,30)=1.0(p>0.05), \mathrm{F}_{\mathrm{CBZ}}{ }^{*}$ event*treatment $(3,30)=1.8(p>0.05)$, respectively. The F-values of the effects of LCM on L-glutamate and ATP were $\mathrm{F}_{\mathrm{LCM}}(3,30)=9.4(p<0.01), \mathrm{F}_{\text {event }}(1,10)=286.3$ $(p<0.01), \mathrm{F}_{\text {treatment }}(1,10)=0.3(p>0.05), \mathrm{F}_{\mathrm{LCM}^{*} \text { treatment }}(3,30)=2.4(p>0.05), \mathrm{F}_{\text {event }} *$ treatment $(1,10)=$ $6.4(p<0.01), \mathrm{F}_{\mathrm{LCM}^{*} \text { event }}(3,30)=1.3(p>0.05)$, and $\mathrm{F}_{\mathrm{LCM}^{*} \text { event }}{ }^{*}$ treatment $(3,30)=2.3(p>0.05)$, and on ATP were $\mathrm{F}_{\mathrm{LCM}}(3,30)=11.3(p<0.01), \mathrm{F}_{\text {event }}(1,10)=441.7(p<0.01), \mathrm{F}_{\text {treatment }}(1,10)=0.8(p>0.05)$, $\mathrm{F}_{\mathrm{LCM}^{*} \text { treatment }}(3,30)=3.9(p<0.05), \mathrm{F}_{\text {event }}{ }^{*}$ treatment $(1,10)=6.6(p<0.05), \mathrm{F}_{\mathrm{LCM}^{*} \text { event }}(3,30)=5.5(p<0.01)$, and $\mathrm{F}_{\mathrm{LCM}^{*} \text { event }}{ }^{*}$ treatment $(3,30)=8.8(p<0.01)$, respectively.

The acute and subchronic administration of ZNS $(30,100$, or $300 \mu \mathrm{M})$ inhibited the astroglial release of both L-glutamate and ATP induced by the first and second FCHK-ACSF-evoked stimulations in 
a concentration-dependent manner (Figure 5A,D). CBZ (10, 30, or $100 \mu \mathrm{M})$ also inhibited the astroglial release of both L-glutamate and ATP induced by the first and second FCHK-ACSF-evoked stimulations (Figure 5C,F). The acute and subchronic administration of therapeutic-relevant concentration of CBZ $(10$ or $30 \mu \mathrm{M})$ did not affect the repetitive FCHK-ACSF-evoked release of L-glutamate and ATP; however, the acute and subchronic administration of a supratherapeutic concentration of CBZ $(100 \mu \mathrm{M})$ inhibited

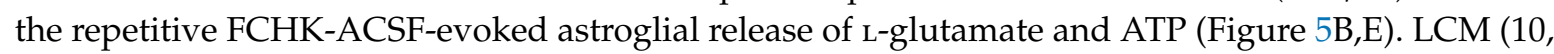
30 , or $100 \mu \mathrm{M}$ ) also inhibited the astroglial release of both L-glutamate and ATP induced by the first and second FCHK-ACSF-evoked stimulations (Figure 5C,F). The acute administration of LCM (10, 30, or $100 \mu \mathrm{M}$ ) did not affect the repetitive FCHK-ACSF-evoked astroglial release of L-glutamate and ATP, whereas the subchronic administration of LCM $(10,30$, or $100 \mu \mathrm{M})$ inhibited the repetitive FCHK-ACSF-evoked astroglial release of L-glutamate and ATP (Figure 5C,F).

Therefore, both the acute and the subchronic administration of therapeutic-relevant or supratherapeutic concentration of CBZ, LCM, and ZNS did not affect the astroglial release associated with unactivated hemichannels; however, CBZ, LCM, and ZNS exhibited distinct targets regarding the astroglial release associated with hemichannels.

\subsection{Concentration-Dependent Effects of the Subchronic Administration of LCM, CBZ, and ZNS on Cx43 Expression in the Plasma Membrane Fraction of Primary Cultured Astrocytes (Study_4)}

The Cx43 expression of hepatic and cardiac cells can be decreased by $2 \%$ dimethyl sulfoxide and $4 \%$ ethanol, respectively [33,34]. In the present study, the levels of dimethyl sulfoxide and ethanol in fDMEM for the subchronic administration of $100 \mu \mathrm{M} \mathrm{CBZ}$ and $100 \mu \mathrm{M} \mathrm{LCM}$ were $0.2 \%$ and $1 \%$, respectively. The effects of $0.2 \%$ dimethyl sulfoxide and $1 \%$ ethanol on $\mathrm{Cx} 43$ expression in the plasma membrane fraction of primary cultured astrocytes could not be observed (Supplementary Figure S1).

The subchronic administration (for 7 days) of both a therapeutic-relevant (30 or $100 \mu \mathrm{M}$ ) and a supratherapeutic $(300 \mu \mathrm{M})$ concentration of ZNS decreased the Cx43 expression in the plasma membrane fraction of primary cultured astrocytes in a concentration-dependent manner $(F(3,20)=$ 20.2, $p<0.01)$ (Figure 6). A therapeutic-relevant concentration of CBZ $(30 \mu \mathrm{M})$ did not affect Cx43 expression, but a supratherapeutic concentration of CBZ $(100 \mu \mathrm{M})$ decreased the Cx43 expression in the plasma membrane $(\mathrm{F}(2,15)=7.3, p<0.01)$ (Figure 6). Neither a therapeutic-relevant $(30 \mu \mathrm{M})$ nor a supratherapeutic $(100 \mu \mathrm{M})$ concentration of LCM affected the Cx43 expression in the plasma membrane (Figure 6).

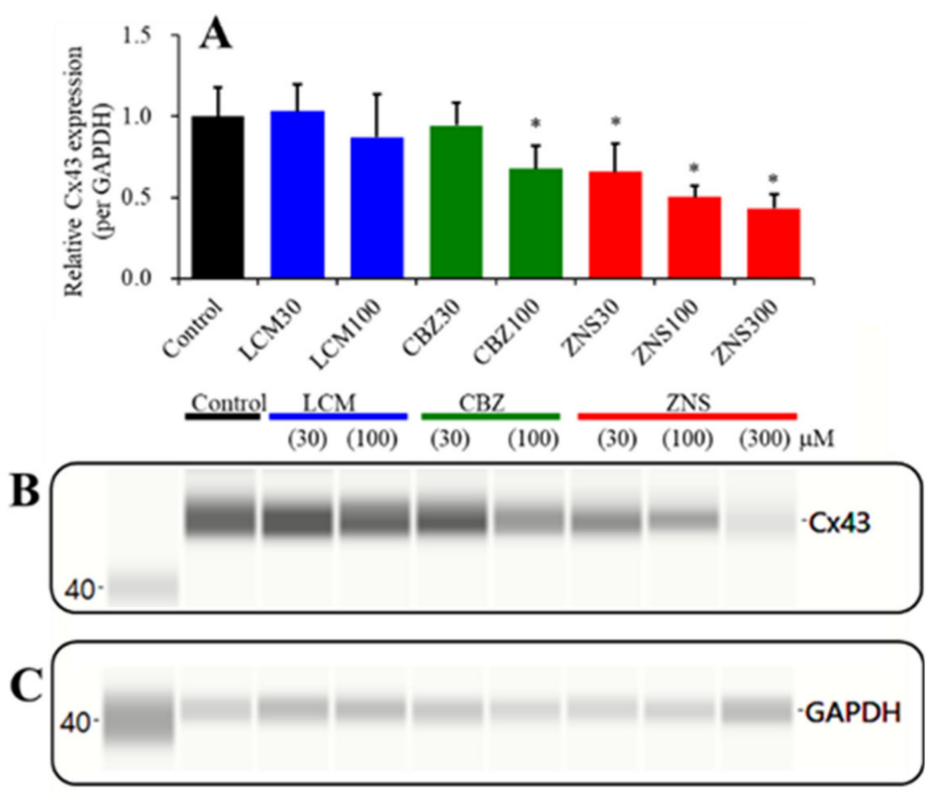

Figure 6. Concentration-dependent effects of the subchronic administration of LCM (30 or $100 \mu \mathrm{M})$, 
CBZ (30 or $100 \mu \mathrm{M})$, and ZNS $(30,100$, or $300 \mu \mathrm{M})$ on the Cx43 expression in the plasma membrane fraction of primary cultured astrocytes (A) and pseudo-gel images using the Simple Western results with anti-Cx43 (B) and anti-GAPDH (C) antibodies for blotting of the plasma membrane fractions. In (A), ordinate: mean $\pm \mathrm{SD}(n=6)$ of the relative protein level of $\mathrm{Cx} 43 .{ }^{*} p<0.05$ relative to the control by one-way ANOVA with Tukey's multiple comparison.

\subsection{Effects of the Extracellular $\mathrm{K}^{+}$and $\mathrm{Ca}^{2+}$ Levels on the Release of $\mathrm{L}-$ Glutamate and ATP in the Orbitofrontal Cortex (OFC)}

To study of the regulation mechanisms of the extracellular cations on the release of L-glutamate and ATP, the present study determined the effects of perfusion with modified Ringer's solution (MRS) containing $50 \mathrm{mM} \mathrm{K}^{+}$(MK-MRS), $100 \mathrm{mM} \mathrm{K}^{+}$(HK-MRS), and $\mathrm{Ca}^{2+}$-free with $100 \mathrm{mM} \mathrm{K}^{+}$(FCHK-MRS) on the extracellular levels of L-glutamate and ATP in the OFC using microdialysis. The perfusion medium was commenced with MRS or MRS containing $0.1 \mu \mathrm{M}$ PSB12379 (ectoATPase inhibitor) for L-glutamate and ATP, respectively. When the coefficients of variation for L-glutamate and ATP reached $<5 \%$ over a period of $60 \mathrm{~min}$ (stabilization), control data were obtained over another $60 \mathrm{~min}$ period (pretreatment period), and then the perfusion medium was switched to MK-MRS, HK-MRS, or FCHK-MRS for $20 \mathrm{~min}$.

The extracellular L-glutamate levels in the OFC were increased by the extracellular $\mathrm{K}^{+}$levels in a concentration-dependent manner $\left(\mathrm{F}_{\text {Time }}(1.4,21.3)=209.5, p<0.01\right) ; \mathrm{F}_{\text {ion }}(2,15)=10.4, p<0.01 ;$ and $\mathrm{F}_{\text {Time }}{ }^{*}$ ion $\left.(2.8,21.3)=21.3, p<0.01\right)$ (Figure 7A,B). The area under curve (AUC) values of the L-glutamate release induced by HK-MRS and FCHK-MRS were almost equal (Figure 7B); however, the L-glutamate release induced by FCHK-MRS (depletion of $\mathrm{Ca}^{2+}$ ) decreased in the initial phase (20-40 min), but increased in the late phase (120-180 $\mathrm{min}$ ) of the L-glutamate release compared to the HK-MRS-evoked release (Figure 7A). The extracellular ATP levels in the OFC were increased by the extracellular $\mathrm{K}^{+}$ levels in a concentration-dependent manner $\left(\mathrm{F}_{\text {Time }}(3.2,48.5)=67.7, p<0.01 ; \mathrm{F}_{\text {ion }}(2,15)=14.0, p<0.01\right.$; and $\mathrm{F}_{\text {Time }}{ }^{*}$ ion $\left.(6.5,48.5)=11.4, p<0.01\right)$ (Figure 7C,D). Furthermore, depletion of extracellular $\mathrm{Ca}^{2+}$ enhanced the $\mathrm{K}^{+}$-evoked ATP release (Figure 7C,D).
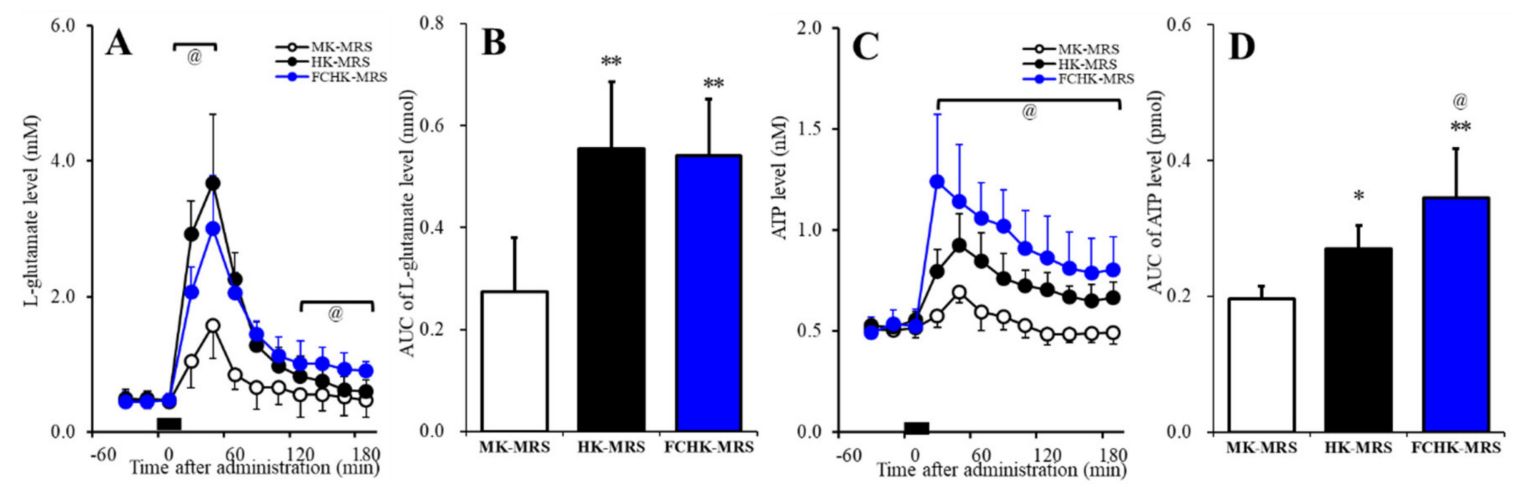

Figure 7. Effects of perfusion with $50 \mathrm{mM} \mathrm{K}^{+}$containing modified Ringer's solution (MRS) (MK-MRS), $100 \mathrm{mM} \mathrm{K}^{+}$containing MRS (HK-MRS), and $\mathrm{Ca}^{2+}$-free with $100 \mathrm{mM} \mathrm{K}^{+}$containing MRS (FCHK-MRS) on the extracellular levels of (A,B) L-glutamate and (C,D) ATP in the orbitofrontal cortex (OFC). Ordinates: the mean extracellular levels of L-glutamate $(\mu \mathrm{M})$ and ATP $(n M)(n=6)$; abscissas: time after perfusion with MK-MRS, HK-MRS, or FCHK-MRS for 20 min (black bars). The area under the curve (AUC) values of the extracellular levels of L-glutamate (nmol) and ATP (pmol) after perfusion with MK-MRS, HK-MRS, or FCHK-MRS during 20-180 min of (A,B) are represented in (C,D), respectively. ${ }^{*} p<0.05$ and ${ }^{* *} p<0.01$ relative to MK-MRS, and ${ }^{\circledR} p<0.05$ relative to HK-MRS by MANOVA with Tukey's post-hoc test. 
2.6. Effects of the Subchronic Administration of a Therapeutic-Relevant Dose of CBZ, LCM, and ZNS on the Repetitive FCHK-MRS-Evoked Release of $\mathrm{L}$-Glutamate and ATP in the OFC

The results using primary cultured astrocytes (Study_1 Study_4) indicate that repetitive FCHK-ACSF-evoked stimulation is a candidate astroglial activation technique. Furthermore, in our previous study, local administration of ZNS and the Cx43 selective inhibitor GAP19 prevented the repetitive HK-MRS-evoked L-glutamate release through activated astroglial hemichannels in the secondary motor cortex [8]. Based on the results of our previous study and Study_1 Study_4 in this report, to study the effects of the subchronic administration of a therapeutic-relevant dose of ZNS, CBZ, and LCM on the release of L-glutamate and ATP in the OFC, rats were subchronically administrated ZNS, CBZ, and LCM (0 or $25 \mathrm{mg} / \mathrm{kg} /$ day for 7 days) using a subcutaneous osmotic pump (2ML_1, Alzet) [9].

Repetitive FCHK-MRS stimulation increased the extracellular levels of L-glutamate and ATP (Figure 8A,E and Figure 9A,B) in the OFC in a use-dependent manner. After the 240 min recovery from the first FCHK-MRS-evoked stimulation, the basal extracellular levels of L-glutamate and ATP were higher than the basal levels of L-glutamate and ATP before the first FCHK-MRS stimulation (first vs. second in Figure 8A,E and Figure 9A,B). The second FCHK-MRS-evoked stimulation also increased the release of L-glutamate and ATP (Figure 8A,E and Figure 9A,B). The release of L-glutamate and ATP induced by the second FCHK-MRS-evoked stimulation was larger than those by the first FCHK-MRS-evoked stimulation (Figure 8A,E and Figure 9A,B).

Subchronic administration of a therapeutic-relevant dose of ZNS (25 mg/kg/day) for 7 days inhibited the repetitive FCHK-MRS-evoked release of L-glutamate and ATP in the OFC (Figure 8B,F and Figure 9A,B). The differences of the basal extracellular levels of L-glutamate and ATP before and after the first FCHK-MRS-evoked stimulation could not be detected in rats administrated ZNS subchronically (Figure 8B,F and Figure 9A,B). The differences between the release of L-glutamate and ATP induced by the first and the second FCHK-MRS-evoked stimulations also could not be detected in rats administrated ZNS subchronically (Figure 8B,F and Figure 9A,B).

Subchronic administration of a therapeutic-relevant dose of CBZ ( $25 \mathrm{mg} / \mathrm{kg} /$ day $)$ for 7 days inhibited the release of both L-glutamate and ATP induced by repetitive FCHK-MRS-evoked stimulation in the OFC (Figures 8C and 9A,B). Subchronic CBZ administration decreased the elevation of the basal levels of L-glutamate and ATP after the first FCHK-MRS-evoked stimulation compared to the control rats, whereas the basal extracellular levels of L-glutamate and ATP after the second FCHK-MRS-evoked stimulation became larger than those before the first stimulation (Figure 8C,G and Figure 9A,B).

Subchronic administration of a therapeutic-relevant dose of LCM ( $25 \mathrm{mg} / \mathrm{kg} /$ day) for 7 days inhibited the release of L-glutamate and ATP induced by repetitive FCHK-MRS-evoked stimulation in the OFC (Figure 8D,H and Figure 9A,B). Subchronic LCM administration decreased the elevation of the basal levels of L-glutamate and ATP after the first FCHK-MRS-evoked stimulation compared to the control rats, whereas the basal extracellular levels of L-glutamate and ATP after the first FCHK-MRS-evoked stimulation became larger than those before the first stimulation (Figure $8 \mathrm{D}, \mathrm{H}$ and Figure 9A,B). 

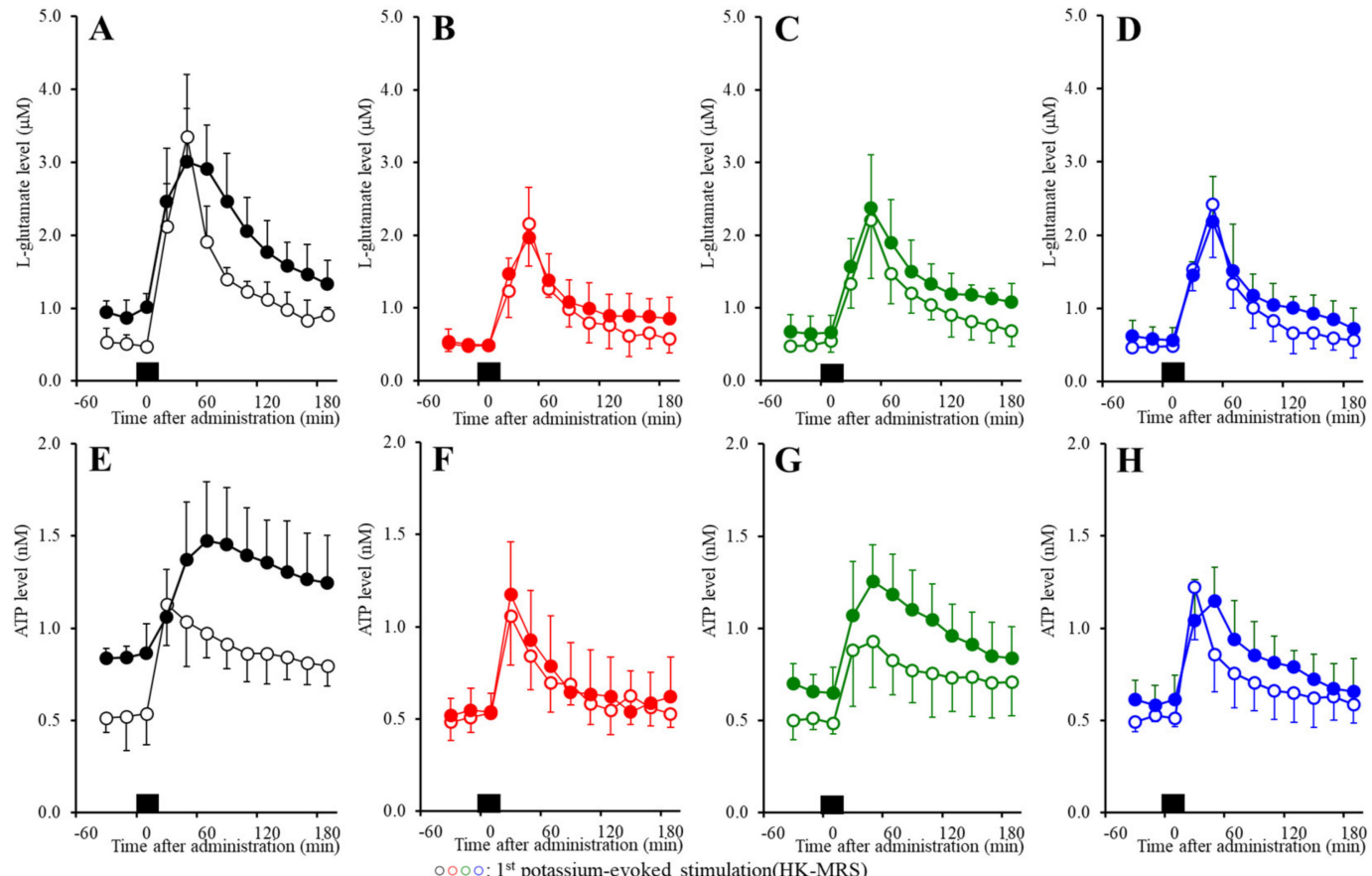

Figure 8. Effects of the subchronic administration of a therapeutic-relevant dose of $(\mathbf{B}, \mathbf{F}) \mathrm{ZNS},(\mathbf{C}, \mathrm{G})$ CBZ, and (D,H) and LCM $\left(25 \mathrm{mg} / \mathrm{kg} /\right.$ day for 7 days) on the repetitive $\mathrm{K}^{+}$-evoked (first stimulation: opened circles; second stimulation: closed circles) release of (A-D) L-glutamate and (E-H) ATP in the OFC. Rats were subchronically administrated with a therapeutic-relevant dose of anticonvulsants. Perfusion medium in the OFC was commenced with MRS. After the stabilization of the levels of L-glutamate and ATP in the OFC, the perfusion medium was switched from MRS to FCHK-MRS for $20 \mathrm{~min}$ (first stimulation). After the first $\mathrm{K}^{+}$-evoked stimulation, the perfusate was returned to MRS for $240 \mathrm{~min}$ (recovery). Following recovery, the perfusate was switched to FCHK-MRS for $20 \mathrm{~min}$ again (second stimulation). After the second FCHK-MRS stimulation, the perfusate was returned to MRS again. Ordinates: mean extracellular levels of L-glutamate $(\mu \mathrm{M})$ and $\operatorname{ATP}(n M)(n=6)$; abscissas: time after the first or second FCHK-MRS stimulations (min). Black bars indicate the perfusion with FCHK-MRS (first and second $\mathrm{K}^{+}$-evoked stimulation).
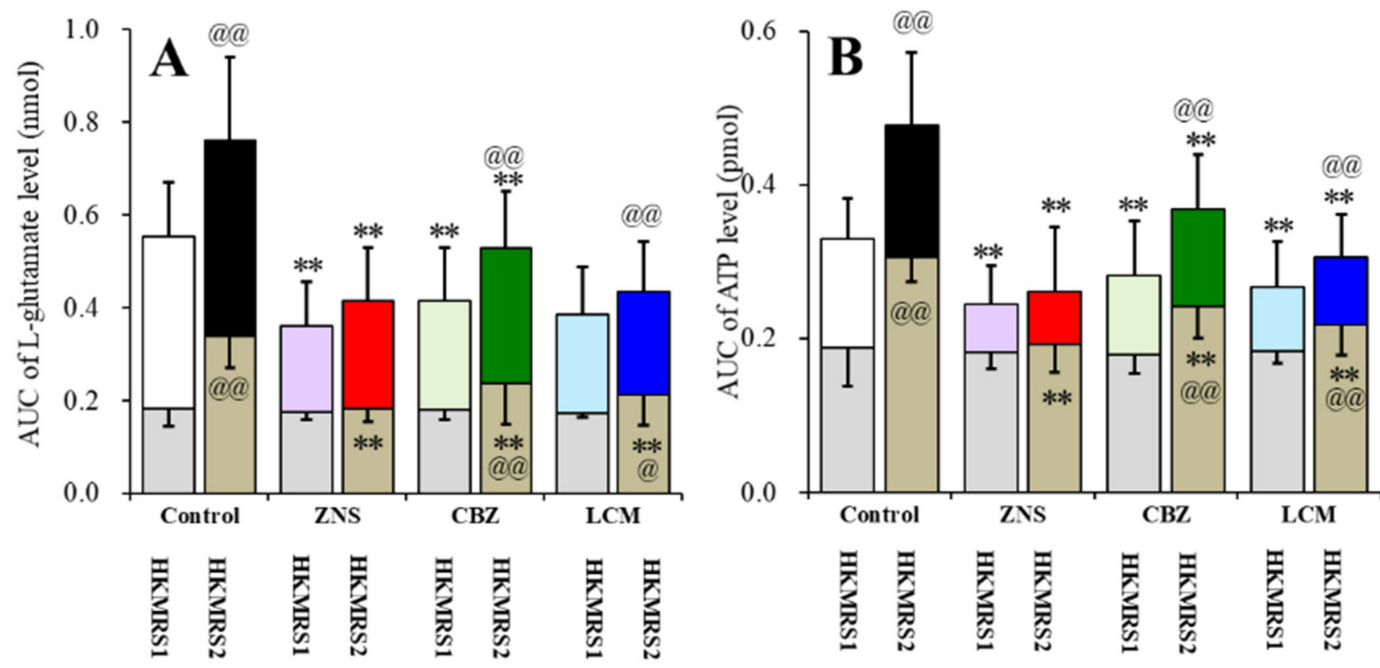

Figure 9. Effects of the subchronic administration of a therapeutic-relevant dose of ZNS, CBZ, and LCM ( $25 \mathrm{mg} / \mathrm{kg} /$ day for 7 days) on the repetitive FCHK-MRS-evoked (first stimulation: HKMRS1; 
second stimulation: HKMRS2) release of (A) L-glutamate and (B) ATP in the OFC. Ordinates: mean AUC values of the extracellular levels of L-glutamate (nmol) and ATP (pmol) before (basal extracellular L-glutamate levels) and after $\mathrm{K}^{+}$-evoked stimulation (from 20 to $180 \mathrm{~min}$ ) of Figure 1 . Gray and colored columns indicate the AUC values of the basal and $\mathrm{K}^{+}$-evoked releases, respectively. ${ }^{*} p<0.05$ and ** $p<0.01$; relative to the control, ${ }^{@} p<0.05$ and ${ }^{\circledR @} p<0.01$ relative to HKMRS (first simulation) by MANOVA with Tukey's post-hoc test. The F-values of the effects of ZNS on L-glutamate and ATP were $\mathrm{F}_{\mathrm{ZNS}}(1,200)=185.0(p<0.01), \mathrm{F}_{\text {time }}(9,200)=53.4(p<0.01), \mathrm{F}_{\text {event }}(1,10)=47.0(p<0.01)$, $\mathrm{F}_{\mathrm{ZNS}{ }^{*} \text { time }}(9,200)=3.7(p<0.01), \mathrm{F}_{\mathrm{ZNS} \text { event }}(1,200)=16.9(p<0.01), \mathrm{F}_{\text {event }}{ }^{*}$ time $(9,200)=2.3(p<0.05)$, and $\mathrm{F}_{\mathrm{ZNS}}{ }^{*}$ event*time $(9,200)=1.1(p>0.05)$, and on ATP were $\mathrm{F}_{\mathrm{ZNS}}(1,200)=216.2(p<0.01), \mathrm{F}_{\text {time }}(9,200)$ $=11.5(p<0.01), \mathrm{F}_{\text {event }}(1,10)=70.5(p<0.01), \mathrm{F}_{\text {ZNS*time }}(9,200)=4.8(p<0.01), \mathrm{F}_{\text {ZNS*event }}(1,200)=$

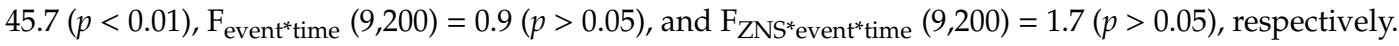
The F-values of the effects of CBZ on L-glutamate and ATP were $\mathrm{F}_{\mathrm{CBZ}}(1,200)=76.9(p<0.01), \mathrm{F}_{\text {time }}$ $(9,200)=50.4(p<0.01), \mathrm{F}_{\text {event }}(1,10)=63.2(p<0.01), \mathrm{F}_{\text {CBZ*time }}(9,200)=2.5(p<0.05), \mathrm{F}_{\text {CBZ }}$ event $(1,200)$ $=5.7(p<0.05), \mathrm{F}_{\text {event*time }}(9,200)=1.8(p>0.05)$, and $\mathrm{F}_{\mathrm{CBZ}}{ }^{*}$ event*time $(9,200)=1.0(p>0.05)$, and on ATP were $\mathrm{F}_{\mathrm{CBZ}}(1,200)=57.6(p<0.01), \mathrm{F}_{\text {time }}(9,200)=11.4(p<0.01), \mathrm{F}_{\text {event }}(1,10)=138.4(p<0.01)$,

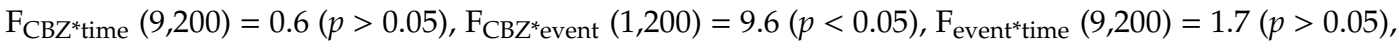
$\mathrm{F}_{\mathrm{CBZ}}{ }^{*}$ event*time $(9,200)=1.1(p>0.05)$, respectively. The F-values of the effects of LCM on L-glutamate and ATP were $\mathrm{F}_{\mathrm{LCM}}(1,200)=147.8(p<0.01), \mathrm{F}_{\text {time }}(9,200)=58.0(p<0.01), \mathrm{F}_{\text {event }}(1,10)=44.2(p<0.01)$, $\mathrm{F}_{\mathrm{LCM}^{*} \text { time }}(9,200)=2.2(p<0.05), \mathrm{F}_{\mathrm{LCM}^{*} \text { event }}(1,200)=16.5(p<0.01), \mathrm{F}_{\text {event }}{ }^{*}$ time $(9,200)=2.7(p<0.01)$, and $\mathrm{F}_{\mathrm{CBZ}}$ event $^{*}$ time $(9,200)=0.8(p>0.05)$, and on ATP were $\mathrm{F}_{\mathrm{LCM}}(1,200)=148.3(p<0.01), \mathrm{F}_{\text {time }}(9,200)$ $=13.8(p<0.01), \mathrm{F}_{\text {event }}(1,10)=103.5(p<0.01), \mathrm{F}_{\text {LCM }^{*} \text { time }}(9,200)=3.8(p<0.05), \mathrm{F}_{\text {LCM }^{*} \text { event }}(1,200)=$ $35.6(p<0.01), \mathrm{F}_{\text {event }}^{*}$ time $(9,200)=3.2(p<0.01)$, and $\mathrm{F}_{\mathrm{LCM}^{*} \text { event }}{ }^{*}$ time $(9,200)=0.7(p>0.05)$, respectively.

\section{Discussion}

\subsection{Regulation Mechanisms of the Astroglial Gliotransmitter Release Associated with Cx43-Containing Hemichannels}

The present study demonstrated various components of event-related astroglial gliotransmitter release and their specific regulation mechanisms using in vivo microdialysis and in vitro primary cultured astrocytes. It has been established that neurotransmitter exocytosis is generated by the $\mathrm{Ca}^{2+}$-dependent exocytosis process, which is activated by elevation of the $\mathrm{Ca}^{2+}$ levels in the presynaptic active zone via inflow through the voltage-sensitive $\mathrm{Ca}^{2+}$ channel or ionotropic glutamate receptor, or output from the endoplasmic reticulum $[6,35,36]$. In the present study, during the resting stage, astroglial hemichannels had an essentially low opening probability similar to previous reports $[8,22,23,30]$, since the hemichannel inhibitors CBX and GAP19 did not affect the astroglial release of L-glutamate and ATP during incubation in ACSF (Figure 1). Contrary to neurotransmitter exocytosis, astroglial hemichannel opening requires depolarization, increased extracellular $\mathrm{K}^{+}$, and/or decreased extracellular $\mathrm{Ca}^{2+}[8,22,23,30]$. In the present study, reduced extracellular $\mathrm{Ca}^{2+}$ alone (FC-ACSF) did not affect astroglial release, but accelerated astroglial release was induced by increased extracellular $\mathrm{K}^{+}$ (FCHK-ACSF), which was suppressed by the hemichannel inhibitors, CBX and GAP19, contrary to basal release (Figure 1). Therefore, depletion of extracellular $\mathrm{Ca}^{2+}$ does not act as a generator, but as an accelerator for astroglial release through activated hemichannels. Taken together with previous electrophysiological studies, the potential for activation of hemichannel activity is probably as follows: depolarization $>$ elevation of extracellular $\mathrm{K}^{+}>$depletion of extracellular $\mathrm{Ca}^{2+}$.

It is well known that elevation of the levels of extracellular $\mathrm{K}^{+}$generates depolarization; however, the sensitivity of neurotransmission and gliotransmission to extracellular $\mathrm{K}^{+}$is different. Indeed, neurotransmitter exocytosis is activated by $25 \mathrm{mM} \mathrm{K} \mathrm{K}^{+}$-evoked stimulation, whereas astroglial release is not increased by $50 \mathrm{mM} \mathrm{K} \mathrm{K}^{+}$-evoked stimulation [30,37]. This discrepancy of $\mathrm{K}^{+}$sensitivity between neurotransmission and gliotransmission is caused by the lower VDSC expression density in non-epileptic astrocytes [38]. Taken together with the previous findings, the present results can lead to the candidate hypothesis for transmission abnormalities between the neurotransmission and gliotransmission associated with activated hemichannel. Toxic hyperexcitable events such 
as epileptic seizures generate an elevation of extracellular $\mathrm{K}^{+}$and a depletion of extracellular $\mathrm{Ca}^{2+}[39,40]$. The depletion of the levels of extracellular $\mathrm{Ca}^{2+}$ suppresses the propagation of epileptic hyperexcitability in attenuation of neurotransmission [39]; however, at the same time, the combination of astroglial depolarization with extracellular ionic abnormality induced by epileptic hyperexcitability activates astroglial hemichannels [39]. The present results support the transition of major components for excitability propagation from neurotransmission to gliotransmission during the sustained hyperexcitable stage. Study of both microdialysis and primary cultured astrocytes demonstrated that repetitive FCHK-MRS-evoked and FCHK-ACSF-evoked stimulations increase the release of L-glutamate and ATP through activated hemichannels in a use-dependent manner (Figure 2, Figure 3, and Figure 9). Therefore, during the initial phase of an epileptic seizure, epileptic hyperexcitability is propagated by $\mathrm{Ca}^{2+}$-dependent neurotransmitter exocytosis, whereas during the late phase, the major propagation components probably shift from neurotransmission to astroglial transmission due to extracellular ionic abnormality. Furthermore, the activation state of astroglial hemichannels is persistent over several hours [8], and the gliotransmitter release through astroglial hemichannels is enhanced and persistent in a use-dependent manner (Figures 2 and 3). These prolonged and use-dependent hyperexcitabilities associated with astroglial transmission contribute to the development of event-related ictogenesis $[8,13,17]$.

\subsection{Inhibitory Effects of the VDSC-Inhibiting Acticonvulsants CBZ, LCM, and ZNS on Activated Cx43 Hemichannels}

We have already demonstrated that the inhibitory effects of ZNS on the Cx43 expression in the plasma membrane fraction play important roles in the inhibition of CBZ-resistant partial epileptic seizures of the genetic ADSHE model S286L-TG [8]. In the present study, astroglial hemichannels are suggested to be activated by toxic hyperexcitable depolarization and its induced depletion of extracellular $\mathrm{Ca}^{2+}$ with elevation of the level of extracellular $\mathrm{K}^{+}$. Based on these findings, before experiment, we speculated that CBZ prevented the repetitive FCHK-MRS-evoked release of L-glutamate and ATP using in vivo microdialysis, but did not affect the repetitive FCHK-ACSF-evoked astroglial release of L-glutamate and ATP using primary cultured astrocytes. The results in this study were in partial agreement with our expectations but could also suggest the more complex mechanisms of ZNS, CBZ, and LCM.

In the microdialysis study, the subchronic administration of therapeutic-relevant dose of CBZ, LCM, and ZNS reduced the repetitive FCHK-MRS-evoked release of L-glutamate and ATP compared to the control (Figures 8 and 9). The use-dependent potentiation of the repetitive FCHK-MRS-evoked release was abolished by ZNS, but was observed in rats subchronically administrated CBZ and LCM (Figure 8B,F and Figure 9A,B). Furthermore, elevation of the basal extracellular levels of L-glutamate and ATP after FCHK-MRS-evoked stimulation, which are released through activated hemichannels [8], was observed in CBZ- and LCM-administrated rats (Figure 8B,F and Figure 9A,B). These results suggest that subchronic administration of therapeutic-relevant dose of CBZ and LCM can prevent propagation of epileptic discharges via their VDSC inhibition but cannot completely prevent activation of astroglial hemichannels. This discrepancy between CBZ, LCM, and ZNS in terms of the repetitive FCHK-MRS-evoked release is induced by two possibilities, a dose-dependent factor or a direct effect on astroglial hemichannels, including activity and hemichannel expression.

In order to clarify the mechanisms of the discrepancy between CBZ, LCM, and ZNS, we determined the acute and subchronic effects of the three anticonvulsants on astroglial hemichannel activity, as well as the subchronic effects of the three anticonvulsants on the Cx43 expression in the astroglial plasma membrane using primary cultured astrocytes. During the resting stage (incubation in ACSF), the astroglial release of L-glutamate and ATP was not affected by acute or subchronic administration of CBZ, LCM, or ZNS (Figure 4). ZNS acutely and subchronically inhibited the first and second FCHK-ACSF-evoked astroglial release in a concentration-dependent manner, but subchronic ZNS predominantly inhibited this release compared to acute administration (Figure 5A,D), possibly caused 
by reduced Cx43 expression in the plasma membrane (Figure 6). Thus, ZNS acutely inhibits astroglial hemichannel activation directly, with a subchronic reduction of functional hemichannels. Contrary to ZNS, neither acute nor subchronic administration of therapeutic-relevant concentration of CBZ $(10$ or $30 \mu \mathrm{M})$ affected the repetitive FCHK-ACSF-evoked astroglial release, whereas both acute and subchronic administration of supratherapeutic concentration of CBZ $(100 \mu \mathrm{M})$ could inhibit it (Figure 5B,E). Furthermore, the inhibitory effects of subchronic administration of supratherapeutic CBZ concentration were predominant compared to acute administration. Indeed, the Cx43 expression in the plasma membrane was decreased and was not affected by supratherapeutic and therapeutic-relevant concentration of CBZ, respectively (Figure 6). Thus, therapeutic-relevant dose/concentration of CBZ could not suppress activation of astroglial hemichannels directly. These differences between ZNS and CBZ can explain the pathophysiology of CBZ-resistant/ZNS-sensitive seizures of S286L-TG [7,8,10]. In our previous study, we speculated that the mechanisms of ZNS-specific effects of astroglial hemichannels are modulated by the enhancement of ubiquitin ligase, the metabotropic glutamate receptor, or inhibition of carbonic anhydrase [7,8,10,20,41,42]. In order to clarify the specific mechanism of the effects of ZNS on astroglial hemichannel expression in the plasma membrane, we shall demonstrate in further studies in the future.

Subchronic LCM administration reduced the repetitive FCHK-ACSF-evoked astroglial release in a concentration-dependent manner without affecting $\mathrm{Cx} 43$ expression, but acute administration did not affect the repetitive FCHK-ACSF-evoked astroglial release (Figure 5C,F and Figure 6). The mechanisms of the inhibitory effects of LCM on the astroglial release associated with hemichannels are different from those of ZNS but can be evaluated to be within the same targets. Inhibition of collapsin response mediator protein 2 (CRMP2) is considered to be a candidate target regarding the antiepileptic action of LCM, in addition to VDSC inhibition [43]. CRMP2 is a positive mediator against VDSC, the voltage-sensitive $\mathrm{Ca}^{2+}$ channel, and neurite outgrowth [43]. The interaction between LCM or CRMP2 and connexin remains to be clarified, but LCM might inhibit the astroglial hemichannel activation process via CRMP2. Recently, the effectiveness of LCM on CBZ-resistant ADSHE seizures with CHRNA4 mutation was reported [18]. In other words, subchronic LCM administration directly suppresses the function of activated astroglial hemichannel activity without affecting hemichannel expression. Based on the effects of ZNS and LCM on astroglial hemichannels demonstrated in this study, the suppressive action of gliotransmission associated with astroglial hemichannels leads to novel reasonable add-on medication strategies.

Finally, the present study demonstrated the distinct effects between CBZ, LCM, and ZNS on astroglial transmission associated with activated hemichannels; however, this study has several limitations. Firstly, the concentration-dependent effects of the subchronic administration of CBZ, LCM, and ZNS on the Cx43 expression in the plasma membrane fraction of primary cultured astrocytes was demonstrated, but connexin 26 and connexin 30 are also expressed in astrocytes [21]. Unfortunately, we could not obtain suitable antibodies of connexin 26 and connexin 30 for the Simple Western system. We should have screened the antibodies of connexin 26 and connexin 30 to clarify the effects of the anticonvulsant on expressions of connexin 26 and connexin 30 in astrocytes. Secondly, the present study indicated that repetitive stimulation using FCHK-MRS and FCHK-ACSF is a pharmacologically useful technique for the activation of astroglial hemichannels. During repetitive and prolonged hyperexcitability, such as epileptic discharge, the levels of extracellular $\mathrm{K}^{+}$increase and the levels of extracellular $\mathrm{Ca}^{2+}$ decrease; however, FCHK-ACSF and FCHK-MRS are more drastic conditions compared to the pathological toxic hyperactivation state. To clarify the pathomechanisms of epileptic seizures associated with extracellular cation mobilization, we should conduct a more detailed study to determine the concentration-dependent effects of the levels of extracellular $\mathrm{Ca}^{2+}$ and $\mathrm{K}^{+}$in the lower range using epileptic animal models. 


\section{Materials and Methods}

\subsection{Experimental Animals}

The animal care procedures, experimental procedures, and protocols for animal experiments were approved by the Animal Research Ethics Committee of the Mie University School of Medicine (No. 24-35-R3). All studies involving animals have been reported in accordance with the ARRIVE guidelines for reporting experiments involving animals [44]. A total of 66 adult rats were used in the microdialysis study, and 30 neonatal rats were used for the primary cultured astrocytes studies.

\subsection{Preparation of the Primary Cultured Astrocytes}

The astrocytes were prepared using a protocol adapted from previously described methods [20, 30,35,45]. Pregnant Sprague-Dawley rats (SLC, Sizuoka, Japan), which were housed individually in cages, were kept in air-conditioned rooms (temperature, $22 \pm 2{ }^{\circ} \mathrm{C}$ ) set to a $12 \mathrm{~h}$ light/dark cycle, with free access to food and water. The cultured astrocytes were prepared from cortical astrocyte cultures of neonatal Sprague-Dawley rats $(n=30)$ sacrificed by decapitation at $0-24 \mathrm{~h}$ of age $[24,45]$. The cerebral hemispheres were removed under a dissecting microscope. Tissue was chopped into fine pieces using scissors, and then triturated briefly with a micropipette. The suspension was filtered using $70 \mu \mathrm{m}$ nylon mesh (BD, Franklin Lakes, NJ, USA), and then centrifuged. The pellets were then re-suspended in 10 mL Dulbecco's modified Eagle's medium containing 10\% fetal calf serum (fDMEM), which was repeated three times. After culture for 14 days (DIV14), the contaminated cells were removed by shaking in a standard incubator for $16 \mathrm{~h}$ at $200 \mathrm{rpm}$. On DIV21, the astrocytes were removed from the flasks by trypsinization, and then seeded directly onto translucent polyethylene terephthalate (PET) membrane $(1.0 \mu \mathrm{m})$ with 24 -well plates $(\mathrm{BD})$ at a density of $1 \times 10^{5}$ cells $/ \mathrm{cm}^{2}$ for the experiments. From DIV21 to DIV28, the culture medium (fDMEM) was changed twice a week, and CBZ $(0,10,30$, or $100 \mu \mathrm{M}$ containing $0.2 \%$ dimethyl sulfoxide as a vehicle), $\operatorname{LCM}(0,10,30$, or $100 \mu \mathrm{M}$, containing $1 \%$ ethanol as a vehicle), or ZNS $(0,30,100$, or $300 \mu \mathrm{M})$ was added for subchronic administration (for 7 days).

On DIV28, the cultured astrocytes were washed out using artificial cerebrospinal fluid (ACSF), and this was repeated three times. The ACSF comprised $\mathrm{NaCl} 150.0 \mathrm{mM}, \mathrm{KCl} 3.0 \mathrm{mM}, \mathrm{CaCl}_{2} 1.4 \mathrm{mM}$, $\mathrm{MgCl}_{2} 0.8 \mathrm{mM}$, and glucose $5.5 \mathrm{mM}$, buffered to $\mathrm{pH} 7.3$ with $20 \mathrm{mM}$ HEPES buffer [30]. To study the effects of a decrease in the level of extracellular $\mathrm{Ca}^{2+}\left(\mathrm{Ca}^{2+}\right.$-free), an increase in the level of $\mathrm{K}^{+}$ ( $\mathrm{K}^{+}$-evoked stimulation), and both complex conditions $\left(\mathrm{Ca}^{2+}\right.$-free with $\mathrm{K}^{+}$-evoked stimulation) on the astroglial release of $\mathrm{L}$-glutamate and ATP, the cultured astrocytes were incubated in $\mathrm{Ca}^{2+}$-free ACSF (FC-ACSF), ACSF containing $100 \mathrm{mM} \mathrm{K}^{+}$(HK-ACSF), or $\mathrm{Ca}^{2+}$-free with $100 \mathrm{mM} \mathrm{K}^{+}$ion ACSF (FCHK-ACSF) for $20 \mathrm{~min}$ [30]. The ionic composition was modified, and isotonicity was maintained by an equimolar decrease of sodium ions $[9,30]$.

\subsection{Simple Western Analysis}

Simple Western analyses were performed using Wes (ProteinSimple, Santa Clara, CA, USA) according to the ProteinSimple user manual $[7,30]$. The lysate of the primary cultured astrocytes was mixed with a master mix (ProteinSimple) to a final concentration of $1 \times$ sample buffer, $1 \times$ fluorescent molecular weight markers, and $40 \mathrm{mM}$ dithiothreitol, and then heated at $95^{\circ} \mathrm{C}$ for $5 \mathrm{~min}$. The samples, blocking reagent, primary antibodies, horseradish peroxidase (HRP)-conjugated second antibodies, chemiluminescent substrate, and separation and stacking matrices were also dispensed to designated wells in a 25-well plate. After plate loading, the separation electrophoresis and immunodetection steps took place in the capillary system and were fully automated. The Simple Western analyses were carried out at room temperature, and the instrument's default settings were used. Capillaries were first filled with the separation matrix, followed by the stacking matrix and about $40 \mathrm{~nL}$ sample loading. During electrophoresis, the proteins were separated on the basis of molecular weight through the stacking and separation matrices at 250 volts for $40-50 \mathrm{~min}$, and then immobilized on the capillary wall using 
proprietary photo-activated capture chemistry. The matrices were then washed out. The capillaries were next incubated with a blocking reagent for $15 \mathrm{~min}$, and the target proteins were immunoprobed with primary antibodies followed by HRP-conjugated secondary antibodies (Anti-Rabbit Detection Module, DM-001, ProteinSimple). The antibodies of GAPDH (NB300-322SS, Novus Biologicals, Littleton, CO, USA) and Cx43 (C6219, Sigma-Aldrich, St. Louis, MO, USA) were diluted in antibody diluent (ProteinSimple) at a 1:100 dilution. The antibody incubation time was 0-120 min with antibody diluents. Luminol and peroxide (ProteinSimple) were then added to generate chemiluminescence, which was captured by a charge-coupled device (CCD) camera. The digital image was analyzed with Compass software (ProteinSimple), and the quantified data of the detected protein were reported as molecular weight and signal/peak intensity.

\subsection{Preparation of the Microdialysis System}

Sprague-Dawley rats (SLC, Shizuoka, Japan) were housed in individual cages in air-conditioned rooms (temperature $22 \pm 2{ }^{\circ} \mathrm{C}$ ) with a $12 \mathrm{~h}$ light/dark cycle and ad libitum access to food and water. After the subchronic administration of $25 \mathrm{mg} / \mathrm{kg} /$ day CBZ, LCM, and ZNS for 7 days using a subcutaneously osmotic pump (2ML_1, Alzet, Cupertino, CA), the rats were anesthetized with 1.8\% isoflurane and then placed in a stereotactic frame [46-48]. A concentric direct insertion-type dialysis probe (0.22 $\mathrm{mm}$ diameter, $3 \mathrm{~mm}$ exposed membrane; Eicom, Kyoto, Japan) was implanted in the orbitofrontal cortex (OFC: $\mathrm{A}=+3.2 \mathrm{~mm}, \mathrm{~L}=+2.4 \mathrm{~mm}, \mathrm{~V}=-6.5 \mathrm{~mm}$, relative to bregma) $[49,50]$.

Perfusion experiments were started $18 \mathrm{~h}$ after recovery from isoflurane anesthesia. The perfusion rate was set at $2 \mu \mathrm{L} / \mathrm{min}$ in all experiments using modified Ringer's solution (MRS) composed of the following (in $\mathrm{mM}$ ): $\mathrm{NaCl}$ (145.0), $\mathrm{KCl}$ (2.7), $\mathrm{CaCl}_{2}$ (1.2), and $\mathrm{MgCl}_{2}$ (1.0), buffered with $2 \mathrm{mM}$ phosphate buffer and $1.1 \mathrm{mM}$ Tris buffer at $\mathrm{pH}$ 7.4. To determine the effects of the levels of extracellular $\mathrm{K}^{+}$and $\mathrm{Ca}^{2+}$, the perfusion medium was changed from MRS to $50 \mathrm{mM} \mathrm{K}{ }^{+}$containing MRS (MK-MRS): $\mathrm{NaCl}$ (97.7), $\mathrm{KCl}$ (50.0), $\mathrm{CaCl}_{2}$ (1.2), and $\mathrm{MgCl}_{2}$ (1.0); $100 \mathrm{mM} \mathrm{K}{ }^{+}$containing $\mathrm{MRS}$ (HK-MRS): $\mathrm{NaCl}$ (47.7), $\mathrm{KCl}$ (100.0), $\mathrm{CaCl}_{2}$ (1.2), and $\mathrm{MgCl}_{2}$ (1.0); and $\mathrm{Ca}^{2+}$-free with $100 \mathrm{mM} \mathrm{K}{ }^{+}$containing MRS (FCHK-MRS): $\mathrm{NaCl}$ (49.1), $\mathrm{KCl}$ (100.0), and $\mathrm{MgCl}_{2}$ (1.0), buffered with $2 \mathrm{mM}$ phosphate buffer and $1.1 \mathrm{mM}$ Tris buffer at $\mathrm{pH} 7.4$ for $20 \mathrm{~min}$ [51-53]. To determine the levels of extracellular L-glutamate and ATP, MRS with and without $0.1 \mu \mathrm{M}$ PSB12379 (ectoATPase inhibitor) was perfused, respectively. When the coefficients of variation for L-glutamate and ATP reached $<5 \%$ over a period of $60 \mathrm{~min}$ (stabilization), control data were obtained over another $60 \mathrm{~min}$ period (pretreatment period), followed by switching to MK-MRS, HK-MRS, or FCHK-MRS for 20 min.

To explore the effects of the subchronic administration of a therapeutic-relevant dose of CBZ, LCM, and ZNS on astroglial hemichannel-associated transmitter release in the OFC induced by repetitive FCHK-MRS-evoked stimulations, the perfusion medium was commenced with MRS. After stabilization of the levels of L-glutamate and ATP, the perfusate was switched to FCHK-MRS for 20 min (the first FCHK-MRS-evoked stimulation). After the first stimulation, the perfusate was returned to MRS for $240 \mathrm{~min}$ (recovery). After the recovery, the perfusate was switched to FCHK-MRS (the second stimulation) for $20 \mathrm{~min}$. After the second stimulation, the perfusate was returned to MRS again.

Following the microdialysis experiments, the rats were anesthetized under $1.8 \%$ isoflurane, and their brains were removed. The locations of the dialysis probes were verified by histological examination using 200- $\mu$ m-thick tissue slices (Vibratome 1000, Technical Products International, St. Louis, MO).

\subsection{Ultra-High-Performance Liquid Chromatography (UHPLC)}

The level of L-glutamate was determined using ultra-high-performance liquid chromatography (UHPLC) equipped with xLC3185PU (Jasco, Tokyo, Japan) and fluorescence detection (xLC3120FP, Jasco), following dual derivatization with isobutyryl-L-cysteine and $o$-phthalaldehyde [54,55]. The derivatization solutions were prepared by dissolving isobutyryl-L-cysteine $(2 \mathrm{mg})$ and o-phthalaldehyde $(2 \mathrm{mg}$ ) in $0.1 \mathrm{~mL}$ ethanol, followed by the addition of $0.9 \mathrm{~mL}$ sodium borate 
buffer (0.2 M, pH 9.0) [35]. Automated pre-column derivatization was performed by drawing $5 \mu \mathrm{L}$ aliquots of the sample, standard, or blank solutions and $5 \mu \mathrm{L}$ of the derivatization solution together into a reaction vial, and then incubating for $5 \mathrm{~min}$ before injection. The derivatized samples $(5 \mu \mathrm{L}$ aliquots) were injected by an autosampler (xLC3059AS, Jasco). The analytical column (YMC Triat C18, particle $1.8 \mu \mathrm{m}, 50 \times 2.1 \mathrm{~mm}$; YMC, Kyoto, Japan) was maintained at $45^{\circ} \mathrm{C}$ and the flow rate was set at $500 \mu \mathrm{L} / \mathrm{min}$. A linear gradient elution program was performed over a period of $10 \mathrm{~min}$ with mobile phases $\mathrm{A}(0.05 \mathrm{M}$ citrate buffer, $\mathrm{pH} 5.0)$ and $\mathrm{B}(0.05 \mathrm{M}$ citrate buffer containing $30 \%$ acetonitrile and $30 \%$ methanol, $\mathrm{pH} 3.5)$. The excitation/emission wavelengths of the fluorescence detector were set at 280/455 nm [56].

\subsection{Ultra-High-Performance Liquid Chromatography with Mass Spectrometry (LCMS)}

The levels of ATP were determined by UHPLC (ACQUITY UPLC H-Class system; Waters, Milford, MA, USA) with mass spectrometry (Acquity SQ detector; Waters). Twenty microliters of filtrated samples were injected using an autosampler (ACQUITY UPLC Sample Manager FTN; Waters). The concentrations of ATP were separated by UHPLC equipped with a Hypercarb column (particle $3 \mu \mathrm{m}, 150 \times 2.1 \mathrm{~mm}$; Thermo, Waltham, MA, USA) at $35^{\circ} \mathrm{C}$, and the mobile phase was set at $450 \mu \mathrm{L} / \mathrm{min}$. A linear gradient elution program was performed over $10 \mathrm{~min}$ with mobile phases A ( $1 \mathrm{mM}$ ammonium acetate buffer, $\mathrm{pH} 11$ ) and $\mathrm{B}$ (acetonitrile). The nitrogen flows of the desolvation and cone were set at 750 and $5 \mathrm{~L} / \mathrm{h}$, respectively, and the desolvation temperature was set at $450{ }^{\circ} \mathrm{C}$. The cone voltage for the determination of ATP $(\mathrm{m} / \mathrm{z}=508.2)$ was $34 \mathrm{~V}$.

\subsection{Chemical Agents}

The non-selective hemichannel inhibitor carbenoxolone (CBX) [30,32] and the selective Cx43 inhibitor GAP19 [30,32] were obtained from Funakoshi (Tokyo, Japan). The ecto-5'-nucleotidase (ectoATPase) inhibitor N6-Benzyl- $\alpha, \beta$-methyleneadenosine 5'-diphosphate disodium salt (PSB12379) was obtained from Namiki (Tokyo, Japan). Lacosamide (LCM) was also obtained from Funakoshi. Carbamazepine (CBZ) was obtained from Tokyo Chemical Industry (Tokyo, Japan). Zonisamide sodium salt (ZNS) was provided by Dainippon-Sumitomo Pharma (Osaka, Japan).

All compounds were prepared on the day of the experiment. CBZ was initially dissolved at $50 \mathrm{mM}$ in dimethyl sulfoxide; he final concentration of dimethyl sulfoxide was lower than $0.2 \%(v / v)$. LCM was initially dissolved at $10 \mathrm{mM}$ in ethanol; the final concentration of ethanol was lower than $1.0 \%$ $(v / v)$. GAP19, CBX, PSB12379, and ZNS were directly dissolved in ACSF or fDMEM for the primary cultured astrocyte study.

The therapeutic-relevant concentrations of CBZ, ZNS, and LCM were 17-42 $\mu \mathrm{M}$ [57-59], $47-330 \mu \mathrm{M}[36,60,61]$, and 20-40 $\mu \mathrm{M}[4,62]$, respectively. Therefore, in the present study, the primary cultured astrocytes were subchronically administrated by CBZ $(10,30$, or $100 \mu \mathrm{M}), \operatorname{LCM}(10,30$, or $100 \mu \mathrm{M})$, and ZNS $(30,100$, or $300 \mu \mathrm{M})$ for 7 days, respectively. According to previous study, the rats were systemically administrated $25 \mathrm{mg} / \mathrm{kg} /$ day CBZ, LCM, and ZNS for 7 days $[4,57,58,63-65]$ using a subcutaneously osmotic pump (2ML_1, Alzet); the nominal pumping rate and duration were $10 \mu \mathrm{L} / \mathrm{h}$ and 7 days.

\subsection{Data Analysis}

All experiments in this study were designed with equally sized animal groups $(n=6)$ without carrying out a formal power analysis, in keeping with previous studies $[7,10,13,30,46,50,66]$. All values are expressed as mean \pm standard deviation (SD), and $p<0.05$ (two-tailed) was considered statistically significant for all tests. The drug levels in the acute local and subchronic administrations were selected based on the values in previous studies $[7,10,13,30,46,50,66,67]$. Where possible, we sought to randomize and blind the data. In particular, for the determination of the extracellular transmitter levels, the sample order on the autosampler was determined by a random number table. 
Data in the microdialysis study were analysed by the Mauchly's sphericity test, followed by multivariate analysis of variance (MANOVA) using BellCurve for Excel ver. 3.2 (Social Survey Research Information Co., Ltd., Tokyo, Japan). When the data did not violate the assumption of sphericity $(p>0.05)$, the $f$-value of the MANOVA was analysed using sphericity-assumed degrees of freedom. However, if the assumption of sphericity was violated $(p<0.05)$, the $f$-value was analysed using Chi-Muller's corrected degrees of freedom. When the F-value for the event/drug/time factors of the MANOVA was significant, the data were analysed by the Tukey's post-hoc test. Data in the astroglial release studies were analysed by using MANOVA or analysis of variance (ANOVA) using BellCurve for Excel ver. 3.2 (Social Survey Research Information Co., Ltd., Tokyo, Japan). If the $f$-value for the event/drug/time factors of the MANOVA or ANOVA was significant, the data were analysed by Tukey's multiple comparison. Data in the Simple Western study were analyzed by one-way analysis of variance (ANOVA) with Tukey's multiple comparison using BellCurve for Excel. All statistical analyses complied with the recommendations on the experimental design and analysis in pharmacology [68].

\subsection{Nomenclature of Targets and Ligands}

The key protein targets and ligands in this article are hyperlinked to the corresponding entries in http://www.guidetopharmacology.org, which is the common portal for data from the IUPHAR/BPS Guide to PHARMACOLOGY [69], and are permanently archived in the Concise Guide to PHARMACOLOGY 2017/18 [70].

\section{Conclusions}

The present study demonstrated the distinct effects of VDSC-inhibiting anticonvulsants CBZ, LCM, and ZNS on gliotransmitter (i.e., L-glutamate and ATP) release through activated astroglial hemichannels. Astroglial hemichannels cannot release gliotransmission at low opening probability during the resting stage; however, plasma membrane depolarization and elevation of extracellular $\mathrm{K}^{+}$with a reduction of extracellular $\mathrm{Ca}^{2+}$ activate astroglial hemichannels, and also generate persistent gliotransmitter release through activated hemichannels. A therapeutic-relevant dose/concentration of CBZ cannot affect astroglial hemichannel activity or Cx43 expression in the plasma membrane. Contrary to CBZ, both ZNS and LCM suppress gliotransmitter release through astroglial hemichannels, but the suppressive mechanisms between ZNS and LCM are distinct. A therapeutic-relevant dose/concentration of ZNS directly inhibits astroglial hemichannel activity with Cx43 expression in the plasma membrane in a concentration-dependent manner, whereas LCM inhibits hemichannel activity without affecting Cx43 expression. Therefore, the present study demonstrated the distinct pharmacological profiles between CBZ, LCM, and ZNS in terms of astroglial transmission associated with activated hemichannels. These results suggest that the distinct effects of these three VDSC-inhibiting anticonvulsants possibly contribute to the mechanisms of their antiepileptic profiles and/or adverse effects.

Supplementary Materials: The following are available online at http://www.mdpi.com/1424-8247/13/6/117/s1, Figure S1: Effects of subchronic administration of dimethyl sulfoxide and ethanol on Cx43 expression in the plasma membrane fraction of primary cultured astrocytes (Study_4).

Author Contributions: Conceptualization, M.O.; Data curation, K.F., Y.U., and M.O.; Formal analysis, K.F. and M.O.; Funding acquisition, M.O.; Methodology, M.F. and M.O.; Project administration; M.O., Validation, Y.U., and M.O.; Writing original draft, M.O. All authors have read and agreed to the published version of the manuscript.

Funding: This study was supported by Japan Society for the Promotion of Science (15H04892 and 19K08073).

Conflicts of Interest: The authors state no conflict of interest.

\section{References}

1. Sills, G.J.; Rogawski, M.A. Mechanisms of action of currently used antiseizure drugs. Neuropharmacology 2020, 168, 107966. [CrossRef] 
2. Meador, K.J.; Loring, D.W.; Boyd, A.; Echauz, J.; LaRoche, S.; Velez-Ruiz, N.; Korb, P.; Byrnes, W.; Dilley, D.; Borghs, S.; et al. Randomized double-blind comparison of cognitive and EEG effects of lacosamide and carbamazepine. Epilepsy Behav. 2016, 62, 267-275. [CrossRef]

3. Arif, H.; Buchsbaum, R.; Weintraub, D.; Pierro, J.; Resor, S.R., Jr.; Hirsch, L.J. Patient-reported cognitive side effects of antiepileptic drugs: Predictors and comparison of all commonly used antiepileptic drugs. Epilepsy Behav. 2009, 14, 202-209. [CrossRef]

4. Rogawski, M.A.; Tofighy, A.; White, H.S.; Matagne, A.; Wolff, C. Current understanding of the mechanism of action of the antiepileptic drug lacosamide. Epilepsy Res. 2015, 110, 189-205. [CrossRef]

5. Ijff, D.M.; Aldenkamp, A.P. Cognitive side-effects of antiepileptic drugs in children. Handb. Clin. Neurol. 2013, 111, 707-718. [CrossRef]

6. Okada, M.; Zhu, G.; Yoshida, S.; Kanai, K.; Hirose, S.; Kaneko, S. Exocytosis mechanism as a new targeting site for mechanisms of action of antiepileptic drugs. Life Sci. 2002, 72, 465-473. [CrossRef]

7. Fukuyama, K.; Fukuzawa, M.; Shiroyama, T.; Okada, M. Pathogenesis and pathophysiology of autosomal dominant sleep-related hypermotor epilepsy with S284L-mutant alpha4 subunit of nicotinic ACh receptor. Br. J. Pharm. 2020, 177, 2143-2162. [CrossRef]

8. Fukuyama, K.; Fukuzawa, M.; Ruri, O.; Okada, M. Upregulated connexin 43 induced by loss-of-functional S284L-mutant alpha4 subunit of nicotinic ACh receptor contributes to pathomechanisms of autosomal dominant sleep-related hypermotor epilepsy. Pharmaceuticals 2020, 13, 58. [CrossRef]

9. Fukuyama, K.; Fukuzawa, M.; Okada, M. Upregulated and hyperactivated thalamic connexin 43 plays important roles in pathomechanisms of cognitive impairment and seizure of autosomal dominant sleep-related hypermotor epilepsy with S284L-mutant $\alpha 4$ subunit of nicotinic ACh receptor. Pharmaceuticals 2020, 13, 99. [CrossRef]

10. Fukuyama, K.; Fukuzawa, M.; Shiroyama, T.; Okada, M. Pathomechanism of nocturnal paroxysmal dystonia in autosomal dominant sleep-related hypermotor epilepsy with S284L-mutant $\alpha 4$ subunit of nicotinic ACh receptor. Biomed Pharm. 2020, 126, 110070. [CrossRef]

11. Shiba, Y.; Mori, F.; Yamada, J.; Migita, K.; Nikaido, Y.; Wakabayashi, K.; Kaneko, S.; Okada, M.; Hirose, S.; Ueno, S. Spontaneous epileptic seizures in transgenic rats harboring a human ADNFLE missense mutation in the beta2-subunit of the nicotinic acetylcholine receptor. Neurosci. Res. 2015, 100, 46-54. [CrossRef]

12. Yamada, J.; Zhu, G.; Okada, M.; Hirose, S.; Yoshida, S.; Shiba, Y.; Migita, K.; Mori, F.; Sugawara, T.; Chen, L.; et al. A novel prophylactic effect of furosemide treatment on autosomal dominant nocturnal frontal lobe epilepsy (ADNFLE). Epilepsy Res. 2013, 107, 127-137. [CrossRef] [PubMed]

13. Zhu, G.; Okada, M.; Yoshida, S.; Ueno, S.; Mori, F.; Takahara, T.; Saito, R.; Miura, Y.; Kishi, A.; Tomiyama, M.; et al. Rats harboring S284L Chrna4 mutation show attenuation of synaptic and extrasynaptic GABAergic transmission and exhibit the nocturnal frontal lobe epilepsy phenotype. J. Neurosci. 2008, 28, 12465-12476. [CrossRef] [PubMed]

14. Tinuper, P.; Bisulli, F.; Cross, J.H.; Hesdorffer, D.; Kahane, P.; Nobili, L.; Provini, F.; Scheffer, I.E.; Tassi, L.; Vignatelli, L.; et al. Definition and diagnostic criteria of sleep-related hypermotor epilepsy. Neurology 2016, 86, 1834-1842. [CrossRef]

15. Scheffer, I.E.; Bhatia, K.P.; Lopes-Cendes, I.; Fish, D.R.; Marsden, C.D.; Andermann, F.; Andermann, E.; Desbiens, R.; Cendes, F.; Manson, J.I.; et al. Autosomal dominant frontal epilepsy misdiagnosed as sleep disorder. Lancet 1994, 343, 515-517. [CrossRef]

16. Provini, F.; Plazzi, G.; Tinuper, P.; Vandi, S.; Lugaresi, E.; Montagna, P. Nocturnal frontal lobe epilepsy. A clinical and polygraphic overview of 100 consecutive cases. Brain 1999, 122, 1017-1031. [CrossRef]

17. Okada, M.; Zhu, G.; Yoshida, S.; Kaneko, S. Validation criteria for genetic animal models of epilepsy. Epilepsy Seizure 2010, 3, 109-120. [CrossRef]

18. Asioli, G.M.; Rossi, S.; Bisulli, F.; Licchetta, L.; Tinuper, P.; Provini, F. Therapy in sleep-related hypermotor epilepsy (SHE). Curr. Treat. Options Neurol. 2020, 22, 1. [CrossRef]

19. Miyajima, T.; Kumada, T.; Saito, K.; Fujii, T. Autism in siblings with autosomal dominant nocturnal frontal lobe epilepsy. Brain Dev. 2013, 35, 155-157. [CrossRef]

20. Fukuyama, K.; Tanahashi, S.; Hoshikawa, M.; Shinagawa, R.; Okada, M. Zonisamide regulates basal ganglia transmission via astroglial kynurenine pathway. Neuropharmacology 2014, 76, 137-145. [CrossRef] 
21. Medina-Ceja, L.; Salazar-Sanchez, J.C.; Ortega-Ibarra, J.; Morales-Villagran, A. Connexins-based hemichannels/channels and their relationship with inflammation, seizures and epilepsy. Int. J. Mol. Sci. 2019, 20, 5976. [CrossRef] [PubMed]

22. Kar, R.; Batra, N.; Riquelme, M.A.; Jiang, J.X. Biological role of connexin intercellular channels and hemichannels. Arch. Biochem. Biophys. 2012, 524, 2-15. [CrossRef] [PubMed]

23. Fasciani, I.; Temperan, A.; Perez-Atencio, L.F.; Escudero, A.; Martinez-Montero, P.; Molano, J.; Gomez-Hernandez, J.M.; Paino, C.L.; Gonzalez-Nieto, D.; Barrio, L.C. Regulation of connexin hemichannel activity by membrane potential and the extracellular calcium in health and disease. Neuropharmacology 2013, 75, 479-490. [CrossRef] [PubMed]

24. Fukuyama, K.; Okada, M. Effects of levetiracetam on astroglial release of kynurenine-pathway metabolites. Br. J. Pharm. 2018, 175, 4253-4265. [CrossRef]

25. He, J.T.; Li, X.Y.; Yang, L.; Zhao, X. Astroglial connexins and cognition: Memory formation or deterioration? Biosci. Rep. 2020, 40. [CrossRef]

26. Hussein, A.M.; Ghalwash, M.; Magdy, K.; Abulseoud, O.A. Beta lactams antibiotic ceftriaxone modulates seizures, oxidative stress and connexin 43 expression in hippocampus of pentylenetetrazole kindled rats. $J$. Epilepsy Res. 2016, 6, 8-15. [CrossRef]

27. Das, A.; Wallace, G.C.T.; Holmes, C.; McDowell, M.L.; Smith, J.A.; Marshall, J.D.; Bonilha, L.; Edwards, J.C.; Glazier, S.S.; Ray, S.K.; et al. Hippocampal tissue of patients with refractory temporal lobe epilepsy is associated with astrocyte activation, inflammation, and altered expression of channels and receptors. Neuroscience 2012, 220, 237-246. [CrossRef]

28. Garbelli, R.; Frassoni, C.; Condorelli, D.F.; Trovato-Salinaro, A.; Musso, N.; Medici, V.; Tassi, L.; Bentivoglio, M.; Spreafico, R. Expression of connexin 43 in the human epileptic and drug-resistant cerebral cortex. Neurology 2011, 76, 895-902. [CrossRef]

29. Li, Q.; Li, Q.Q.; Jia, J.N.; Liu, Z.Q.; Zhou, H.H.; Mao, X.Y. Targeting gap junction in epilepsy: Perspectives and challenges. Biomed. Pharm. 2019, 109, 57-65. [CrossRef]

30. Fukuyama, K.; Okubo, R.; Murata, M.; Shiroyama, T.; Okada, M. Activation of astroglial connexin is involved in concentration-dependent double-edged sword clinical action of clozapine. Cells 2020, 9, 414. [CrossRef]

31. Rusakov, D.A. Depletion of extracellular $\mathrm{Ca}^{2+}$ prompts astroglia to moderate synaptic network activity. Sci. Signal 2012, 5, 4. [CrossRef] [PubMed]

32. Wang, N.; De Bock, M.; Decrock, E.; Bol, M.; Gadicherla, A.; Bultynck, G.; Leybaert, L. Connexin targeting peptides as inhibitors of voltage-and intracellular ${ }^{\mathrm{Ca} 2+}$-triggered $\mathrm{C} \times 43$ hemichannel opening. Neuropharmacology 2013, 75, 506-516. [CrossRef] [PubMed]

33. Maes, M.; Yanguas, S.C.; Willebrords, J.; Vinken, M. Models and methods for in vitro testing of hepatic gap junctional communication. Toxicol. Vitro 2015, 30, 569-577. [CrossRef] [PubMed]

34. Hung, C.L.; Lai, Y.J.; Chi, P.C.; Chen, L.C.; Tseng, Y.M.; Kuo, J.Y.; Lin, C.I.; Chen, Y.C.; Lin, S.J.; Yeh, H.I. Dose-related ethanol intake, Cx43 and Nav1.5 remodeling: Exploring insights of altered ventricular conduction and QRS fragmentation in excessive alcohol users. J. Mol. Cell Cardiol. 2018, 114, 150-160. [CrossRef]

35. Yamamura, S.; Hoshikawa, M.; Dai, K.; Saito, H.; Suzuki, N.; Niwa, O.; Okada, M. ONO-2506 inhibits spike-wave discharges in a genetic animal model without affecting traditional convulsive tests via gliotransmission regulation. Br. J. Pharm. 2013, 168, 1088-1100. [CrossRef] [PubMed]

36. Yoshida, S.; Okada, M.; Zhu, G.; Kaneko, S. Effects of zonisamide on neurotransmitter exocytosis associated with ryanodine receptors. Epilepsy Res. 2005, 67, 153-162. [CrossRef]

37. Kawata, Y.; Okada, M.; Murakami, T.; Mizuno, K.; Wada, K.; Kondo, T.; Kaneko, S. Effects of zonisamide on $\mathrm{K}^{+}$and $\mathrm{Ca}^{2+}$ evoked release of monoamine as well as $\mathrm{K}^{+}$evoked intracellular $\mathrm{Ca}^{2+}$ mobilization in rat hippocampus. Epilepsy Res. 1999, 35, 173-182. [CrossRef]

38. Qiao, X.; Werkman, T.R.; Gorter, J.A.; Wadman, W.J.; van Vliet, E.A. Expression of sodium channel alpha subunits 1.1, 1.2 and 1.6 in rat hippocampus after kainic acid-induced epilepsy. Epilepsy Res. 2013, 106, 17-28. [CrossRef]

39. Carmignoto, G.; Haydon, P.G. Astrocyte calcium signaling and epilepsy. Glia 2012, 60, 1227-1233. [CrossRef]

40. Rusakov, D.; Fine, A. Extracellular $\mathrm{Ca}^{2+}$ depletion contributes to fast activity-dependent modulation of synaptic transmission in the brain. Neuron 2003, 37, 287. [CrossRef] 
41. Omura, T.; Kaneko, M.; Okuma, Y.; Matsubara, K.; Nomura, Y. Endoplasmic reticulum stress and Parkinson's disease: The role of HRD1 in averting apoptosis in neurodegenerative disease. Oxid. Med. Cell Longev. 2013, 2013, 239854. [CrossRef] [PubMed]

42. Yamamura, S.; Hamaguchi, T.; Ohoyama, K.; Sugiura, Y.; Suzuki, D.; Kanehara, S.; Nakagawa, M.; Motomura, E.; Matsumoto, T.; Tanii, H.; et al. Topiramate and zonisamide prevent paradoxical intoxication induced by carbamazepine and phenytoin. Epilepsy Res. 2009, 84, 172-186. [CrossRef] [PubMed]

43. Wilson, S.M.; Khanna, R. Specific binding of lacosamide to collapsin response mediator protein 2 (CRMP2) and direct impairment of its canonical function: Implications for the therapeutic potential of lacosamide. Mol. Neurobiol. 2015, 51, 599-609. [CrossRef] [PubMed]

44. McGrath, J.C.; Lilley, E. Implementing guidelines on reporting research using animals (ARRIVE etc.): New requirements for publication in BJP. Br. J. Pharm. 2015, 172, 3189-3193. [CrossRef] [PubMed]

45. Okada, M.; Fukuyama, K.; Shiroyama, T.; Ueda, Y. Carbamazepine attenuates astroglial L-glutamate release induced by pro-inflammatory cytokines via chronically activation of adenosine A2A receptor. Int. J. Mol. Sci. 2019, 20, 3727. [CrossRef] [PubMed]

46. Okada, M.; Fukuyama, K.; Shiroyama, T.; Ueda, Y. Lurasidone inhibits NMDA antagonist-induced functional abnormality of thalamocortical glutamatergic transmission via 5-HT7 receptor blockade. Br. J. Pharm. 2019, 176, 4002-4018. [CrossRef]

47. Okada, M.; Fukuyama, K.; Okubo, R.; Shiroyama, T.; Ueda, Y. Lurasidone sub-chronically activates serotonergic transmission via desensitization of 5-HT1A and 5-HT7 receptors in dorsal raphe nucleus. Pharmaceuticals 2019, 12, 149. [CrossRef]

48. Okada, M.; Okubo, R.; Fukuyama, K. Vortioxetine subchronically activates serotonergic transmission via desensitization of serotonin 5-HT1A receptor with 5-HT3 receptor inhibition in rats. Int. J. Mol. Sci. 2019, 20, 6235. [CrossRef]

49. Paxinos, G.; Watson, C. The Rat Brain: In Stereotoxic Coordinates, 6th ed.; Academic Press: San Diego, CA, USA, 2007.

50. Okada, M.; Fukuyama, K.; Kawano, Y.; Shiroyama, T.; Suzuki, D.; Ueda, Y. Effects of acute and sub-chronic administrations of guanfacine on catecholaminergic transmissions in the orbitofrontal cortex. Neuropharmacology 2019, 156, 107547. [CrossRef]

51. Kawata, Y.; Okada, M.; Murakami, T.; Kamata, A.; Zhu, G.; Kaneko, S. Pharmacological discrimination between effects of carbamazepine on hippocampal basal, $\mathrm{Ca}^{2+}$ - and $\mathrm{K}^{+}$-evoked serotonin release. Br. J. Pharm. 2001, 133, 557-567. [CrossRef]

52. Okada, M.; Wada, K.; Kiryu, K.; Kawata, Y.; Mizuno, K.; Kondo, T.; Tasaki, H.; Kaneko, S. Effects of Ca ${ }^{2+}$ channel antagonists on striatal dopamine and DOPA release, studied by in vivo microdialysis. Br. J. Pharm. 1998, 123, 805-814. [CrossRef] [PubMed]

53. Okada, M.; Kawata, Y.; Mizuno, K.; Wada, K.; Kondo, T.; Kaneko, S. Interaction between $\mathrm{Ca}^{2+}, \mathrm{K}^{+}$, carbamazepine and zonisamide on hippocampal extracellular glutamate monitored with a microdialysis electrode. Br. J. Pharm. 1998, 124, 1277-1285. [CrossRef] [PubMed]

54. Mtui, E.; Gruener, G.; Dockery, P. Fitzgerald's Clinical Neuroanatomy and Neuroscience, 7th ed.; Elsevier: Philadelphia, PA, USA, 2015.

55. Karlsen, A.S.; Korbo, S.; Uylings, H.B.; Pakkenberg, B. A stereological study of the mediodorsal thalamic nucleus in Down syndrome. Neuroscience 2014, 279, 253-259. [CrossRef] [PubMed]

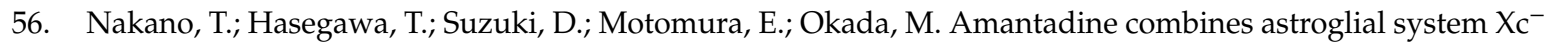
activation with glutamate/NMDA receptor inhibition. Biomolecules 2019, 9, 191. [CrossRef]

57. Okada, M.; Hirano, T.; Mizuno, K.; Chiba, T.; Kawata, Y.; Kiryu, K.; Wada, K.; Tasaki, H.; Kaneko, S. Biphasic effects of carbamazepine on the dopaminergic system in rat striatum and hippocampus. Epilepsy Res. 1997, 28, 143-153. [CrossRef]

58. Okada, M.; Hirano, T.; Mizuno, K.; Kawata, Y.; Wada, K.; Murakami, T.; Tasaki, H.; Kaneko, S. Effects of carbamazepine on hippocampal serotonergic system. Epilepsy Res. 1998, 31, 187-198. [CrossRef]

59. Tanahashi, S.; Yamamura, S.; Nakagawa, M.; Motomura, E.; Okada, M. Effect of lamotrigine and carbamazepine on corticotropin-releasing factor-associated serotonergic transmission in rat dorsal raphe nucleus. Psychopharmacology 2012, 220, 599-610. [CrossRef]

60. Yamamura, S.; Ohoyama, K.; Nagase, H.; Okada, M. Zonisamide enhances delta receptor-associated neurotransmitter release in striato-pallidal pathway. Neuropharmacology 2009, 57, 322-331. [CrossRef] 
61. Masuda, Y.; Utsui, Y.; Shiraishi, Y.; Karasawa, T.; Yoshida, K.; Shimizu, M. Relationships between plasma concentrations of diphenylhydantoin, phenobarbital, carbamazepine, and 3-sulfamoylmethyl-1,2-benzisoxazole (AD-810), a new anticonvulsant agent, and their anticonvulsant or neurotoxic effects in experimental animals. Epilepsia 1979, 20, 623-633. [CrossRef]

62. Greenaway, C.; Ratnaraj, N.; Sander, J.W.; Patsalos, P.N. Saliva and serum lacosamide concentrations in patients with epilepsy. Epilepsia 2011, 52, 258-263. [CrossRef]

63. Wasterlain, C.G.; Stöhr, T.; Matagne, A. The acute and chronic effects of the novel anticonvulsant lacosamide in an experimental model of status epilepticus. Epilepsy Res. 2011, 94, 10-17. [CrossRef] [PubMed]

64. Okada, M.; Kaneko, S.; Hirano, T.; Mizuno, K.; Kondo, T.; Otani, K.; Fukushima, Y. Effects of zonisamide on dopaminergic system. Epilepsy Res. 1995, 22, 193-205. [CrossRef]

65. Okada, M.; Hirano, T.; Kawata, Y.; Murakami, T.; Wada, K.; Mizuno, K.; Kondo, T.; Kaneko, S. Biphasic effects of zonisamide on serotonergic system in rat hippocampus. Epilepsy Res. 1999, 34, 187-197. [CrossRef]

66. Okada, M.; Fukuyama, K.; Kawano, Y.; Shiroyama, T.; Ueda, Y. Memantine protects thalamocortical hyper-glutamatergic transmission induced by NMDA receptor antagonism via activation of system $\mathrm{xc}^{-}$. Pharm. Res. Perspect. 2019, 7, e00457. [CrossRef] [PubMed]

67. Duerrschmidt, N.; Hagen, A.; Gaertner, C.; Wermke, A.; Nowicki, M.; Spanel-Borowski, K.; Stepan, H.; Mohr, F.W.; Dhein, S. Nicotine effects on human endothelial intercellular communication via alpha4beta2 and alpha3beta2 nicotinic acetylcholine receptor subtypes. Naunyn Schmiedebergs Arch. Pharm. 2012, 385, 621-632. [CrossRef] [PubMed]

68. Curtis, M.J.; Alexander, S.; Cirino, G.; Docherty, J.R.; George, C.H.; Giembycz, M.A.; Hoyer, D.; Insel, P.A.; Izzo, A.A.; Ji, Y.; et al. Experimental design and analysis and their reporting II: Updated and simplified guidance for authors and peer reviewers. Br. J. Pharm. 2018, 175, 987-993. [CrossRef]

69. Harding, S.D.; Sharman, J.L.; Faccenda, E.; Southan, C.; Pawson, A.J.; Ireland, S.; Gray, A.J.G.; Bruce, L.; Alexander, S.P.H.; Anderton, S.; et al. The IUPHAR/BPS Guide to pharmacology in 2018: Updates and expansion to encompass the new guide to immunopharmacology. Nucleic. Acids Res. 2018, 46, D1091-D1106. [CrossRef]

70. Alexander, S.P.H.; Christopoulos, A.; Davenport, A.P.; Kelly, E.; Mathie, A.; Peters, J.A.; Veale, E.L.; Armstrong, J.F.; Faccenda, E.; Harding, S.D.; et al. The concise guide to pharmacology 2019/20: G protein-coupled receptors. Br. J. Pharm. 2019, 176, S21-S141. [CrossRef] 